\title{
قياس وتحليل آلية عمل ثلاثية العجز وتوءمة العجز لبلدان منظمة التعاون الاقتصادي باستخدام طريقة العزوم المعقمة (GMM) للمدّة (2017-2009)
}

\author{
أ.د. صباح صابر ممد خوشناو ، قسم الاقتصاد ، جامعة صلاح الدين ، أقليم كوردستان العراق \\ م. هيوا عثمان اساعيل، قسم الاقتصاد ، جامعة صلاح الدين، أقليم كوردستان العراق
}

تكشف هذه الورقة عمل وآلية تقدير فرضية العجز الثلاثي في ضوء تلك التطورات التي طرأت في الاقتصاد العالمي، وتبحث هذه الدراسة تجريبيًا العلاقة بين العجز المالي وججز الحساب الجاري

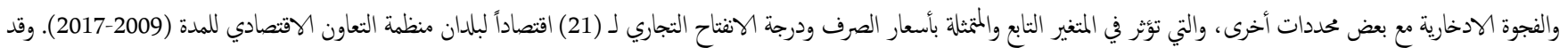

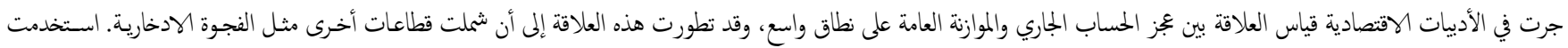

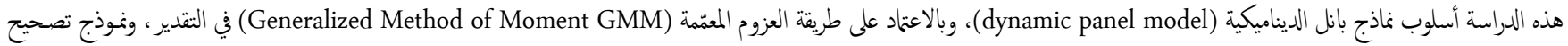

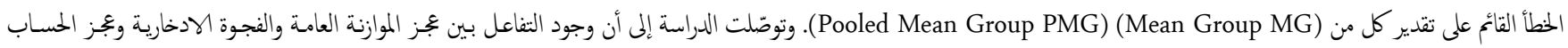

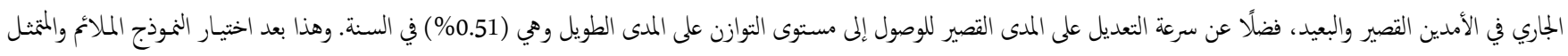

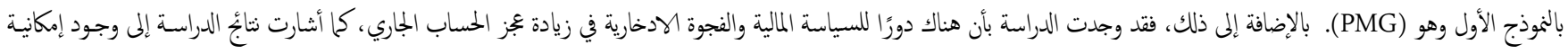

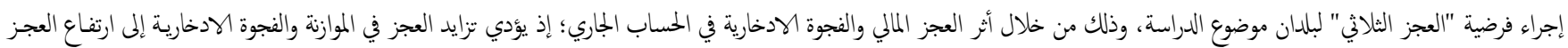

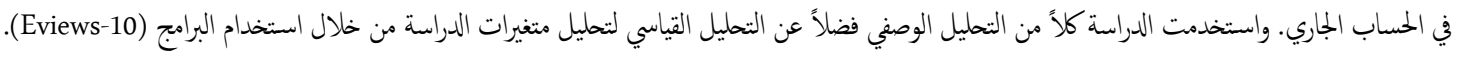
مفاتيح الكلمات: توَمة العجز ، ثلاثية العجز، البيانات اللوحية الديناميكية، بلدان دول منظمة التعاون الاقتصادي، تقدير (GMM)، تقدير (MGG) و (PMG).

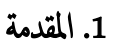

"العجز الثلاثي" من خلال تجربة بعض الدول المتقدمة. وهذه الدراسات كانت تدع البحث والتحقيق عن تلك الآثار التي تتركها هذه الفرضية. ومن هذا المنطلق أُخذ بتجربة اقتصادات بعض الدول المتقدمة، ومن المتوقع أن تساهم في إضافة نظرة جديدة إلى أدبيات الاقتصاد الكلي وبالأخص التوازن الداخلي والخارجي. تنطلق فكرة إدخال هذه الفرضية إلى دراسة التوازن العام من خلال العجز الذي يشهده كل من الموازنة العامة والحساب الجاري، أو ما يسمى بـ "العجز المزدوج"؛ مما انعكس على زيادة الاقتراض للولايات المتحدة الأمريكية وبعض الدول الأخرى المتقدمة من الخارج. ومع ذلك؛ فإن العجز في الحساب الجاري شوّه التدفقات الرأسالية، كما أثرّ هذا التدهور في حساب رأس المال إلى آثار سلبية في الموازنة

العامة وبالتلالي الاختلال في التوازن العام بينها.

1.1 تأتي أهمية الدراسة من خلال الدور الذي يؤديه كل من عجز الموازنة العامة ويجز الحساب الجاري والفجوة الادخارية على كثير من المؤشرات الاقتصادية، وبالثالي إعادة التوازن إلى الاقتصاد على المستويين الداخلي والخارجي. وتبرز أهمية هذه الدراسة بأها نسخة موسعة وأكثر تطورًا من نظرية العجز التوءي، بما في ذلك
يخظى موضوع التوازن الخارجي باهتمام بالغ من قبل العديد من الخبراء الاقتصاديين وصاني السياسة المالية. ويعود هذا الاهتام إلى وجود توثيق وتنسيق شديد بينه وبين التوازن الداخلي، خاصة في ظل اقتصاد ذي أنظمة مثقدمة تعتمد على التقدم التقني والمستجدات العصرية، ورغز أهمية الاستقرار في التوازن الداخلي إلا أنه كنيل للتوازن الخارجي، وتمتد آثاره إلى كافة الأصعدة التي تتعلق بالأنشطة والتبادلات الدولية، ولكنَّ هناك دورًا فعالاً للتوازن الخارجي لإعادة التوازن العام. كان التزكيز الأساسي في هذا المجال على العجز في الموازنة العامة والعجز في الحساب الجاري أو ما يسمى بـ "فرضية العجز المزدوج" فقط، وظهرت هذه الفرضية في الثانينيات نتيجة الارتفاع الحاد في يجز الموازنة العامة في الولايات المتحدة الأمريكة، وبالتالي جاءت آثاره على الحساب الجاري بشكل سلبي، ومع ذلك فإن الظاهرة ليست محصورة فقط في بلد واحد، بل امتدت إلى بلدان أخرى. وكانت الدراسات آنذاك تدور حول تثاثثر السياسات المالية؛ فقد كان من المربح أن تكون غير فعالة كأداة للثأثير على الاقتصاد الحقيقي مع إثارة إلى العجز في الحساب الجاري وكيفية إعادة التوازن بينه وبين التوازن المالي. ومع تطور العلاقات التبادلية والتجارية، ظهر بأن هناك تأثيرًا آخر يسى بـ 


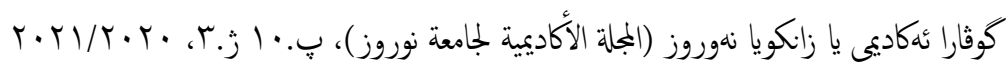

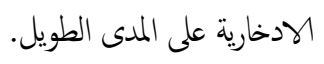

ب. الأثر الإيجابي للموازنة العامة والفجوة الادخرية على الحساب الجاري أو لموبل

$$
\text { تحقيق فرضية ثلاثية العجز. }
$$

ج· تأثير حساب رأس المال في الفجوة الادخارية، وذلك عند عدم كفاية المدخرات المحلية، وزيادة الاستثمار المحلي بسبب التدفق الحر لرأس المال،

وبالتالي يؤدي إلى التسارع في تخقيق التوازن بين الادخار والاستثمار.

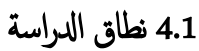

تشمل هذه الدراسة من الناحية المكانية كل من (أستراليا - كندا - دانيارك فلندا - فرنسا - ألمانيا - إسرائيل - إيطاليا - اليابان - هولندا -نيوزيلندابولندا - إسبانيا- السويد- المملكة المتحدة - الولايات المتحدة الأمريكية - سلوفانيا - هنغاريا - بلجيكا - أيسلندا - برتغال) ومن الناحية الزمانية المدة (2009-2017).

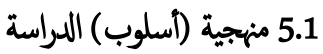

تم الاعتاد في كتابة هذه الدراسة على الجمع بين أسلوبي الاستنبطي والاستقرائي، أي استخدام الأسلوب الوصفي لعينة من بلدان منظمة التعاون الاقتصادي، فضلًا عن استخدام الأسلوب القياسي للمتغرات الأساسية للظاهرة موضوع الدراسة للمدة (2009-2017) الذي يسمح وإجراء واستخدام (طريقة العزوم المعمّمة Generalized Method of Moment GMM) في Pooled PMG/ARDL) التقدير، ونموذج تصحيح الخطأ القائم على تقدير Mean Group ARDL Estimation

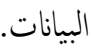

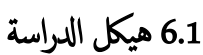

بهدف الوصول إلى أهداف الدراسة قسّمت الدراسة إلى الأجزاء الآتية: المبحث الأول: خخص للإطار النظري لتحليل كل من فرضية "العجز المزدوج" و "العجز الثلاثي". ثُ يستعرض المكونات التجريبية ذات الصلة

$$
\text { بالفرضيات. }
$$

المبحث الثاني: عرض بيانات الدراسة ومنهجيتها الخاصة بالموذج.

$$
\text { المبحث الثالث: يناقش النتائج التجريبية للتحليل القياسي. }
$$

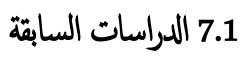

Assessing the ) بعنوان (Shastri \& Mohapatra: 2017) براسة

Triple Deficit Hypothesis for Major South Asian
إدراج وإدخال بعض المفاهيم المهمة إلى هذا التحليل مثل الادخار والاستثمار (صافي التكوين الرأسمالي)، ومن خلاله يككن أن تعمل في إعادة التوازن للاقتصاد

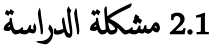

ثكن مشكلة الدراسة في أن أغلب الدراسات المتعلقة بهذا المجال تدور حول العلاقة بين جزز الموازنة العامة ويجز الحساب الجاري مع تجاهل آثار المتغيرات الأخرى على هذه العلاقة، وذلك من خلال تفسير وتحليل فرضيتي (ريكاردو ) و (كينز) في هذا المجال. وهذا بسبب الأسلوب المتبع في تحليل وتفسير هذه الظاهرة، ولكن في أواخر القرن الماضي ظهرت آراء أخرى في إطار تحليل ودراسة بعض المتغيرات الأخرى التي لها آثار على تلك العلاقة الثنائية بين عجز الموازنة العامة والحساب الجاري، وهذه المحاولات لها أهمية كير في صياغة هذه العلاقة في إطار فرضية "العجز الثلاثي".

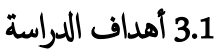

$$
\text { تهدف الدراسة إلى تحقيق ما يلي: }
$$

التعرّف على نسخة أكثر تطورًا من توءمة العجز وتقديم نظرة جديدة بهدف فهم العجز في المدخرات والنكوين الرأسمالي جنبًا إلى جنب مع كل من العجز في الموازنة العامة والعجز في الحساب الجاري وتقييم العلاقة بنهها. دراسة العلاقة بين تيار رصيد الحساب الجاري والموازنة العامة والفجوة الادخارية (الادخار - الاستثار ) ومدى تأثيرها على الاستقرار واستدامة العلاقة بين التوازن الداخلي والخارجي. تحديد النموذج الملائم من بين الناذج القياسية لبيانات الدراسة بهدف الوصول إلى العلاقة الحتيقة بين الموازنة العامة وجزز الحساب الجاري والفجوة الادخارية. ولنلك بعد إجراء كافة الهحدارات لتقدير الناذج القياسية الخختلفة. 4- العمل على خلق مناخ استثاري عالي الكفاءة وتعزيز التعاون والعمل المشترك والتبادل الثقني فيه بين البلدان لغرض الترقي باقتصادات هذه

$$
\text { الدول. }
$$

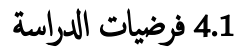

$$
\text { بغرض تحقيق أهداف الدراسة، تفتزض الدراسة ما يأتي: }
$$
أ. وجود علاقة بين جزز الموازنة العامة ويجز الحساب الجاري والفجوة 


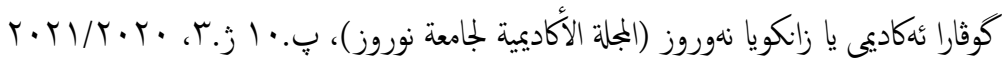

في آسيا للمدة (1985-2012). وتوصّلت الدراسة إلى النتائُ الآتية: - - زيادة العجز الملمي بسب زيادة الإنفاق العام، وهذا يؤدي إلى زيادة

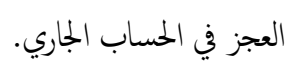

- ميكن أن يُسّن الحساب الجاري من خلال التحكم بأسعار الصرف

$$
\text { وتخنيض قيمة العملة المحلية في البلدان الآسيوية. }
$$

- - تحديد سرعة التعديل على المدى القصير للوصول إلى مستوى

التوازن على المدى الطويل، وتحديد إمكانية تطبيق الفرضيتين

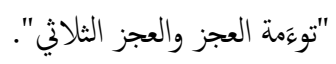

د. دراسة Miscal Deficits Current " بعوان (Miteza, Ilir)

Deficit And Investment: A Panel Causality Framework "Of 20 OECD Countries بين عجز الموازنة وجزز الحساب الجاري، وقد استخدمت الدراسة بيانات اللوحية الديناميكية باستخدام طريقة اللحظات المعمّمة (GMM) للعينة من (20) دولة ذات الاقتصادات المتقدمة للمدة (2008-1974). توصلت الدراسة إلى أن لعجز الموازنة والاستثار الحملي تأثيرًا على الحساب الجاري بحيث يؤدي العجز المستمر والكبير إلى عجز في الحساب الجاري خاصة في المدى القصير ، أما في العجز التوَّم فهناك تأثير في المدى المتوسط، وييدو

$$
\text { أنها تأثير ضعيف في الأجل الطويل. }
$$

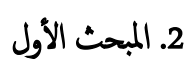

\section{2 الإطار النظري لتحليل فرضية "تومَمة العزز والعجز الثلاثي"}

اكتسبت فرضية "توءم العجز" حظًا وفيرًا من الدراسات في أوائل الثانينيات بعدما شهدت الاقتصاد الأمريكي جزًا كيرًا في الحساب الجاري، وقد رافق ذلك اتساع جزز الموازنة العامة، وبعد ذلك ازداد العجز الخارجي للولايات المتحدة بشكل ملحوظ، إذ أصبحت فرضية العجز المزدوج موضوعًا ساخنًا للدراسات الاقتصادية والسياسات المالية، ومنذ ذلك الوقت كان النقاش يدور حول الصلة بين العجز في الموازنة العامة والحساب الجاري لمدة طويلة، وكذلك السعي لتحقيق الوصول إلى أبعاد تلك العلاقة بشكل مستمر ( Rosensweig

.(\&Tallman:1993,590

وتعود أسباب هذا الاهتمام بهذين المتغيرين إلى خلق الاختلالات في الاقتصاد الكلي والأضرار الاقتصادية على المدى الطويل، وبالتالي نقل الثروة إلى الخارج
(Countries: A Panel Data Analysis العلاقة بين العجز المالي ويجز الحساب الجاري والادخار - الاستثاري لعينة مكونة من (5) دول في جنوب آسيا (1985-2015). وقد استخدمت الدراسة نماذج البانل الديناميكية، وقد أشارت ننائج الدراسة إلى وجود علاقة طوية الأمد بين كل من عزز الموازنة ورصيد الحساب الجاري والفجوة الادخارية، فضلاً عن ذلك؛ فقد حدد الاتجاه والسبية بحيث أن التحسين في الحساب الجاري يتطلب التقشف المالي، بالإضافة إلى أن التحسين في الادخار يؤدي إلى التحسن في الحساب الجاري. ب. براسة (Hilmi \& Fda: 2016) الموسومة بـ " triple deficit or twin divergence: a dynamic panel analysis الدراسة بيان مدى العلاقة بين كل من جزز الموازنة ويزز الحساب الجاري والادخار الخاص من منظور النج الريكاردي والكينزي، وقد وصلت الدراسة إلى وجود علاقة بينها على نطاق واسع، وبرهنت الدراسة إمكنية تطبيق كل من الفرضيتين (توءمة العجز والعجز الثلاثي). وقد استخدمت الدراسة عينة من (24) دولة ذات الاقتصادات المتحولة للمدة (20022013)، واعتمدت على الأسلوب الوصفي والقياسي باستخدام البيانات اللوحية الديناميكية بالاعتماد على طريقة تقنية العزوم المعمّمة (GMM). وخلصت الدراسة بجموعة من الاستنتاجات منها: وجود علاقة إيهابية بين عجز الحساب الجاري ويجز الموازنة العامة وجز المدخرات الاستثمرية، وأثر نسبة الصادرات والواردات على عزز الحساب الجاري وانخفاض عائدات الضرية نتيجة تخفيض الضرائب المباشرة على الواردات. Current Account and " دراسة (Bon:2014)، الموسومة بـ مل Fiscal Deficits Evidence of Twin Divergence from Selected Developing Economies of Asia الدراسة إلى بيان الأسئة المتعلقة حول أثر السياسة المالية والحساب الجاري على الاقتصاد الوطني، وقد بينت الدراسة وجود أثر كل من العجز الملالي وبعض المتغيرات الأخرى مثل التضخم وأسعار الصرف وأسعار الفائدة على الحساب الجاري. وقد اعتمدت الدراسة على الأسلوب القياسي بالاعتماد على طريقة العزوم المعمّمة (GMM) وطريقة نموذج تصحيح الخطأ القائم على تقدير (PMG) لعينة مكونة من (10) اقتصادات للدول النامية 


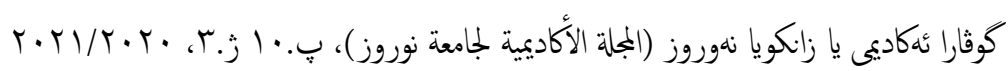

ويكون سوق السلع والخدمات في حالة توازن عندما يكون الإنفاق الخخطط أو

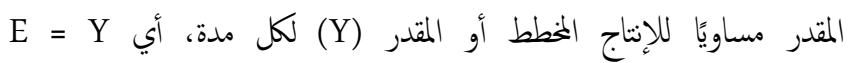
(Raji:2019,94) ( ويعطي هذا التوازن كل من (E=C+I+G+X-M)

(I) الاستهلاك المحلي للسلع والخدمات، ويثل (C=C+SP+T) الاستثخار المحلي ويمثل (G) الإنفاق الحكوي المحلي ويمثل (X) صادرات السلع والخدمات، و يمثل (M) واردات السلع والخدمات، و يمثل (SP) الادخار من القطاع الخاص، و يمثل (T) إجالي الإيرادات الضريبية المستحقة للحكومة المحلية، ويكن إعادة كتابة شرط توازن الدخل بـ (I+G+X=SP+T+M) المدخرات العامة (Sg=T-G)، ويمثل بمجوع الادخار العام والخاص الادخار الوطني أو القوي (Sn) ويترتب على ذلك أن الميزان التجاري يساوي صافي المدخرات الوطنية المخطط لها (Sn-I).

$$
M-X=S^{n}-I \ldots \ldots 1
$$

وإذا أغفلنا توازن الدخل وتوازن الإنفاق الحكوي، فإنه يمكن وصف الحساب التجاري (CA) - (C) بالصيغة الآتية:

$$
\mathrm{CA}=(\mathrm{X}-\mathrm{M}) \ldots \ldots .2
$$

فإذا كانت صادرات الدولة أقل مما تستورد، فإن ذلك يؤدي إلى عز في حسابها الجاري الذي يموَّل من القروض الخارجية (السواعي والعزام: 2015, 101). وفي ضوء هذه المعادلات والعلاقات السابقة ذكرها يككن أن نصل إلى إيياد الادخار القوي من خلال المعادلة النالية (Miteza,Illir:2012,7):

\section{$\mathrm{Sn}=\mathrm{Y}-\mathrm{C}-\mathrm{G}=\mathrm{Sg}+\mathrm{SP} \ldots \ldots \ldots .3$}

على افتراض أن الاقتصاد في حالة تشغيل كامل، فإن المعادلة (1) تعني شرطًا بالنسبة لصافي توازن سوق السلع (M-X)، ويجب أن يوفر الموارد لتغطية الفائض من الإنفاق الاستثاري (I) الذي لا يُوفّر من مصادر محلية الادخار الوطني (Sn). إنه يعكس أي إنفاق محلي أو صادرات لا يكن توفيره من الموارد

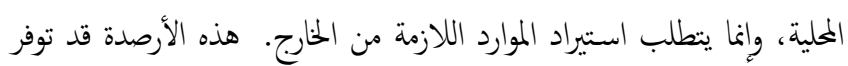
Xملومات مفيدة في عمليات التكيف أو التعديل التي قد تؤثر على الصادرات

$$
\text { (Mt }
$$

الموقف التجاري (X-M) يعادل مجموع صافي الادخار الخاص (Sp-I)، وفائض الموازنة المكومية (X-M) =(SP-I) + (T-G)، تسترعي هذه الصيغة الانتباه إلى العلاقة بين السياسة المالية وأرصدة الحساب الجاري، أي ظاهرة "العجز
والعبء الذي يفرضه على الأجيال القادمة، وفي إطار هذه الدراسات افترضوا أنَّ السبب الأساسي لارتفاع العجز التجاري هو في الواقع تصاعد عجز الموازنة العامة، فضلًا عن ذلك فإنه لا يكن علاج رصيد الحساب الجاري ما لم توضع سياسة

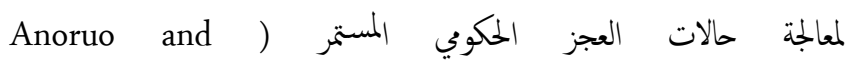
.(Ramchander:1998,487 وتؤكد نظرية "العجز المزدوج" أن العجز في الموازنة العامة والعجز الخارجي في الاقتصاد مترابطان ويؤديان إلى الثدهور في ميزان المدفوعات في نهاية المطاف، وبالتالي إلى تدهور التوازن الخارجي، ويتوقق تطبيق نظرية العجز المزدوج على الواقع الاقتصادي المختلف؛ وذلك بسبب التنوع والاختلاف في الهيكل الاقتصادي لكل منهم، ففي بعض الاقتصادات بمكن تطبيق هذه الفرضية وفي

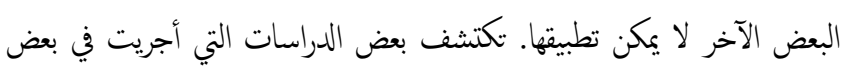
البلدان وأظهرت النتائُ أن هناك علاقة سلبية بين العجز في الموازنة العامة والحساب الجاري، أي وجود أثر سلبي لفرضة العجز والآخر علاقة إيجابية، ومن خلال هذا المبحث نتطرق إلى أهم أبعاد فرضية "توءمة العجز والعجز الثلاثي" لتئي كالنالي: - n

\subsection{2 فرضية "توكمة العجز"} يتعلّق مفهوم "توَهمة العجز بالعجز" في الموازنة العامة والعجز في رصيد الحساب توعي الجاري، وذلك تحت أضواء التطورات الاقتصادية خلال العقدين الأخيرين في القرن الماضي أو الثانينيات من القرن الماضي، قد أدى هذان العجزان (العجز التجاري والعجز المالي) إلى ارتفاع معدلات البطالة، والذي يشار إليه باسم "العجز

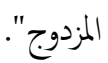
إن الأساس النظري الذي يدع العلاقة بين السياسة المالية والحساب الجاري في ميزان المدفوعات مشتق من خلال منهج الدخل الذي قدمه كل من (مونديل

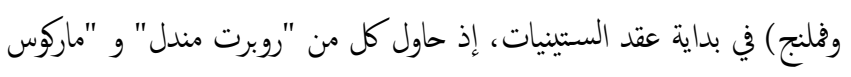
فلنج (Robert Mundell \& Marcus Fleming) توسيع النموذج الكنزي ليشمل الاقتصاد المفتوح بوجود حركة رؤوس أموال؛ فقد نمذجا ميزان المدفوعات لاستخدامه في تحليل فاعلية سياسات الاستقرار الاقتصادي. ويوضح هذا النموذج من خلال منحنى (IS) سوق السلع والخدمات، ومنحنى (LM) سوق النقود، ومنحنى (POB) التوازن في ميزان المدفوعات، وعند تقاطع هذه المنحنيات الثلاثة يكون الاقتصاد في حالة التوازن. 


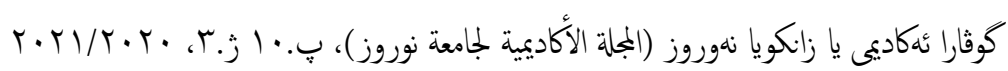

الكلي ونموه إن العجز في الموازنة العامة يطرح مشاكل خطيرة متعلقة بالتوازن الخارجي، وخاصة عندما تكون احتياطات الدولة من العملات تستزف بشكل مستمر نتيجة لناك العجوزات المستمرة؛ مما يؤدي إلى الإفراط في الدين وإعداد المسرح لخلق أزمة اقتصادية أخرى. في حساب ميزان المدفوعات فإن الصورة المتطابقة للحساب الجاري (CA) هي مقياس يعرف باسم "الحساب الرأسمالي والمالي (FA)"، والذي يقيس الندفق الدولي للأصول الرأسمالية، وأن العجز في الحساب الجاري يساوي فائض حساب رأس المال، هذا في حال عدم وجود السهو والخطأ في بيانات ميزان المدفوعات، والذي هو شائع لمعالجة تدفقات رأس المال الدولي، وأن شراء المستثمرين الدوليين الأصول في دولة ما ليس لغرض تمويل العجز في الحساب الجاري لهذه الدولة، وإنما

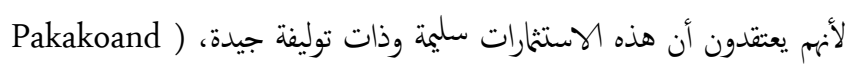
2006,6: poole). وفي هذا المجال نشر (Fausten) ذلك التزابط بين الحسابات الجارية (CA) وحسابات رأس المال (FA) والمعروفة باسم "الحساب المالي"، والتي تعد من المفاهيم الأساسية في الاقتصاد الكلي للاقتصاد المفتوح، ويسسد هذه العلاقة والتزابط بين الحسابات المكونة نتيجة ردود فعل القطاعات المالية والحقيقية على الاضطرابات النظمية والتفاعلية أثناء عملية التعديل أو النكيف (Fausten, 1989). تالك المجموعة من المعادلات التجريبة طوّرت بواسطة قيد ميزان المدفوعات، وأن كلاً من توازن الاقتصاد الكلي المفتوح مدمج في إطار تحليلي مشترك يجدده نوع الاقتصاد المفتوح علاقة نفقات - الدخل، وتتوقق ننائُ هذه العلاقات بناء على نوع البيانات والهيكل الاقتصادي، ويكن أن تكون النتأُج إيجابية أو سلبية، بمعنى أنها تدع هذا التزابط بين الحسابات الجارية والمالية أو

ترفضها (Tang, T. C., \& Fausten: 2012, ويكتب ميزان المدفوعات السابقة بواسطة (BOP=CA+FA=0) وتسمح بالتفسيرات البديلة بأن معاملات الحساب الجاري أو المالم هي مركة الاستعاب (CA=-FA, FA=-CA) قصور في القطاع المال (FA-) بالكامل من خلال فائض الندفقات من الصادرات (M>X) في قطاع السلع والخدمات وبالمثل، يُموَل جزز القطاع الحتيقي (X>M) عن طريق صافي تدفقات رأس المال (FA) أو التدفقات من الخارج. كما لاحظ أن علاقات الدخل والإنفاق تعكس بأن رصيد الحساب (Pakakoand poole)

الجاري (CA=Sn-) يعادل رصيد الادخار والاستثخار الوطني (Sn-I) أي
المزدوج". وبإعادة كتابة شروط الحساب الجاري ورصيد الموازنة العامة مع (T-G) ولى التوالي خصل على الصيغة التالية: (CA=X-M) $\mathrm{CA}=\mathrm{S}^{\mathrm{n}}-\mathrm{I}+\mathrm{PB} \ldots \ldots .4$

في معادلة (4) قد يُعوَّض العجز في الموازنة أو عدم الادخار الحكوي بزيادة المدخرات الماصة أو انخفاض في الاستثمرات المحلية أو الصادرات، وهذا التعديل قد يتسبب في انخفاض الميزان التجاري وفقًا لظاهرة العجز المزدوج. وتعكس فرضية العجز المزدوج أن التوازن الخارجي تهيمن عليه الموازنة العامة، وأن العجز

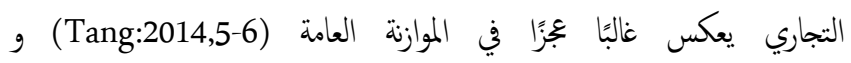
(السواعي :(102, 2015). (Miteza,Illir:2012,7) ويسود الفكر الاقتصادي في هذا المجال منهجان تفسيريان للعلاقة بين عجز الحساب الجاري ويجز الموازنة العامة وهما النكفؤ الريكاردي والمنهج الكينزي. ويبين النكفؤ الريكاردي عدم وجود علاقة بين جزز الحساب الجاري وجزز الموازنة العامة، وتفسير ذلك هو أن اتساع جز الموازنة نتيجة تقليص حجم الضرائب (T) ذو أثر مؤقت لا بد أن تتلاشى آثاره بعودة الضرائب إلى مستوياتها الأصلية. أما المقتح الكينزي؛ فيدعي وجود علاقة مباشرة بين عجز الحساب الجاري ويجز الموازنة، ويحدد اتجاه هذه العلاقة من عجز الموازنة باتجاه كجز الحساب الجاري، وهذا ما يسمى بـ "توكَمة العجزين"، وتفسير ذلك هو أن اتساع عجز الموازنة يأتي نتيجة لزيادة الإنفاق الحكوي، وبارتفاع مستوى الإنفاق الحكوي سينخفض مستوى لئس الادخار الحكوي والقوي، وبانخاض مستوى الادخار القوي ستقل المدخرات ويرتفع معدلات الفائدة، وارتفاع معدلات الفائدة سيزداد من طلب الأجانب على العملة المحلية؛ ما يؤدي إلى ارتفاع في سعر صرف العملة الوطنية مقابل العملات الأجنبية، وبارتفاع سعر صرف العملة الوطنية تصبح الواردات أكثر إغراءً للمواطنين وأقل تكلفة؛ مما يزيد من الواردات، وستصبح الصادرات أقل جذبًا للأجانب وأغلى تكلة؛؛ مما يخفض من الصادرات. ونتيجة لزيادة الواردات وانخفاض الصادرات يتشكل جزز الميزان التجاري. إن الميزان التجاري هو المحدد الرئيس في تغيرات رصيد الحساب الجاري، فإن عجز الحساب الجاري سوف تئرس يتزايد، وبذلك فإنّ يجز الموازنة العامة يؤدي إلى عجز الحساب الجاري. (الكسواني:

.29-28, 2001

\subsection{2 فرضية "العجز المزدوج والعجز الثلاثي"}

إن العجزين الداخلي والخارجي الكيبرين والمستمرين ههدان استقرار الاقتصاد 


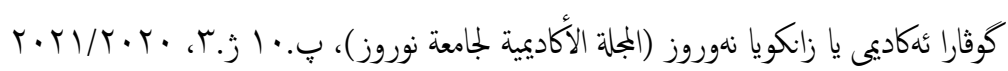

الفجوة الادخارية (الادخار -الاستثار) ويرافقه الاختلال الخارجي المتثلل بالعجز في الحساب الجاري أو العجز التجاري. ويككن القول: إن العجز الداخلي يؤدي إلى العجز في الحساب الجاري، أو هو الذي يدف إلى العجز التجاري. ويضع "ثلاثي العجز" الربط بين كل من التوازن الداخلي المتثل بالموازنة العامة والادخار الاستثمار مع التوازن الخارجي. من أجل توضيح مفهوم "العجز الثلاثي"، وقبل أي شيء؛ من الضروري دراسة كيفية حدوث التوازن الكلي في الاقتصاد؟ ومقابل هذا، هناك مفهوم العجز بمعنى عدم التوازن في المتغيرات الثلاث في الاقتصاد الكلي. في هذه النقطة، إن الأساس النظري والتجريبي لنظرية العجز الثلاثي، والذي فسَّره كينز في متطابقة

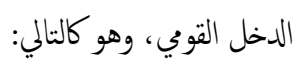

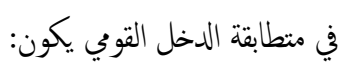

$$
\mathrm{Y}=\mathrm{C}+\mathrm{I}+\mathrm{G}+(\mathrm{X}-\mathrm{M}) \ldots \ldots 6
$$

إذ تثثل (C) الإنغاق الاستهلاكي، وتثثل (I) الإنغاق الاستثفري، و (G) الإنفاق الحكوي، ويدل (X) على الصادرات، ويمثل (M) الواردات السلع والخدمات، و صافي الصادرات. (X-M) والإنفاق الحكوي في الاقتصاد تعد الإنغاق الكلي أو الطلب الكلي وتعبر بالرمز (AE) كما نرى في المعادلة رخ (7) فني الجانب الأيسر في المعادلة كتبت (AE). ونحل على المعادلة رقة (8) نتيجة ترتيب المعادلة رق (7). وبعدها نخصل على

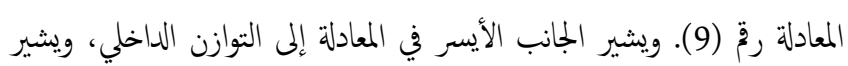

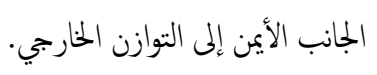

$$
A E=\mathrm{C}+\mathrm{I}+\mathrm{G} \ldots \ldots 7
$$$$
Y=\mathrm{AE}+(\mathrm{X}-\mathrm{M}) \ldots \ldots 8
$$

$$
Y-\mathrm{AE}=(\mathrm{X}-\mathrm{M} \ldots \ldots 9
$$

إذا كان الإنفاق أكبر من الإنتاج في الاقتصاد المفتوح، فهذا يعني أن الجانب الأيسر في المعادلة ذات الإثارة السالبة، وبها يحدث العجز الداخلي في هذا الاقتصاد. وفي هذه الحالة، فإن الجانب الأيمن من المعادلة أيضًا قيمة سالبة لتوفير

$$
\text { التوازن؛ مما يعني حدوث العجز المارجي أيضًا. }
$$

إن مصادر العجز الخارجي التي تنشأ في اقتصاد يكن أن يكون السبب القطاع الخاص والقطاع العام، ويكن تفسير ذلك من خلال التسريبات والحقن في الاقتصاد المفتوح. إن الاستثمار(I)، والإنفاق الحكوي (G)، والصادرات (X)،
I في الاقتصاد المفتوح، ويكن استثار الادخار في الداخل أو في الخارج بحيث

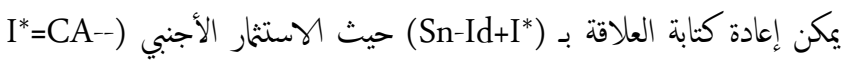
(FA اقتناء الأصول الأجنبية (FA<0) والتحويلات المتناسبة للموارد المحلية الحتيقية في الخارج (CA>0) بحيث: (Tang: 2014,6) (Raji:2019,95). $\left(S^{\mathrm{n}}-\mathrm{I}\right)=\mathrm{CA}=-\mathrm{FA} \ldots \ldots . .5$

\subsection{2 فرضية "ثلاثية العجز"}

تجسد العديد من الدراسات حول "عجز المزدوج أو توَهمة العجز"، وتصنيفها إلى تلك التي تدع فرضية النكافؤ الريكاردو والنهج الكينزي، وأدرجت فرضية "العجز الثلاثي" في جدول أعال سياسة الاقتصاد الكلي الدولي للعديد من البلدان، خاصة بعد التطورات التي طرأت على الواقع الاقتصاد العالمي والتبادلات التجارية الدولية وظهور بعض الاختلالات وإمكانية موابحة هذه الاختلالات من خلال معرفة آثار كل من فرضية توءمة العجز السائد وفرضية ثلاثي العجز الجديدة التي ظهرت في السنوات الأخيرة. وتكون هذه الفرضية امتدادًا لفرضية العجز التوءَم مع إدراج فجوة الادخار. والفكرة الأساسية لهذه الفرضية هي الربط بين الموازنة العامة وتوازن الادخار والاستثار، وبناءً على ذلك فإن العجز في الموازنة العامة إلى جانب العجز في الادخار والاستثمر ، يحفزان جزًَا تجاريًا. وفي هذا المجال يكن أن نشير إلى أبعاد هذه الفرضية بحيث أن في أي اقتصاد إذا زاد كل من العجز في الموازنة العامة والفجوة الادخارية والعجز في الميزان التجاري في نفس المدة الزمنية معًا، فإن ذلك يسمى بـ "الفجوة الثلاثية" في هذا العجز المتزامن كل من القطاع العام أي عدم التوازن في الإيرادات العامة النفقات العامة وبالقطاع الخاص الاختلال في الادخار - الاستثمار في إطار Bayramoglu \& ) التوازن الداخلي يسبب العجز في الحساب الجاري .(Ozturk:2018,212-213 في فرضية توَّمة العجز يتم الاعتماد المتبادل بين كل من عجز الحساب الجاري ويجز الموازنة العامة، وبناءً على هذه الفكرة يككن أن نصل إلى صياغة أخرى لتحليل

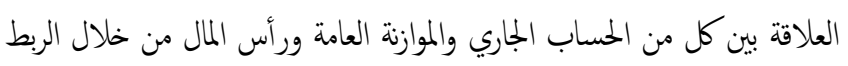
بين العلاقات الثلاث في إطار فرضية "العجز الثلاثي" من منظور التوازن العام مع تطبيق نهج الدخل والنفقات. إن مفهوم "ثلاثي العجز" ينبثق من تدهور الاختلال المحلي المتزامن مع وجود يجز

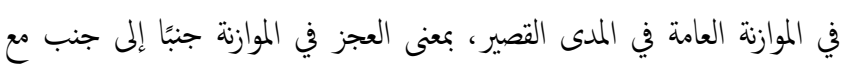




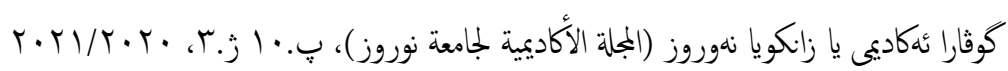

عجز في الموازنة العامة، فني هذه الحالة يكون الاقتصاد في كجز مزدوج. وفجوة الادخار التي تنشأ سبب انخفاض الكفاءة، الاقتصاد المحلي لتلبية الاستثمارات المحلية ويرافقه العجز المزدوج، وبالتالي يخلق العجز ، وبها يضاف جز آخر على العجزيين (Bayramoglu \& Ozturk:2018,212-214). في البلدان منخفضة الدخل فإن ارتفاع مستويات الفقر يجعل من المستحيل تقريبًا توليد وفورات كافية لتوفير الأموال اللازمة لتمويل المشاريع الاستثمارية. وهذا بزيد من العتماد على المعونات المشروطة أو المقيدة. وتعرف هذه المشكلة باسم "جوة الادخار". فاخخفاض معدلات الادخار وضعف الأسواق المالية المتطورة يجعلان من العوائق الكبيرة أمام القطاعين العام والخاص للحصول على الأموال الاستثارية، ويعوق أيضًا ارتفاع تكليف الاقتراض أو عبء الاستثارات الرأسمالية. وتختلف الفجوة الادخارية مع العملات الأجنبية، إذ تعاني الدول الفقيرة من الفجوة الادخارية بسبب عدم وجود مدخرات محلية كافية لكي تتمكن من تمويل المعدل المطلوب من الاستثارات الرأسمالية لتعزيز النمو الاقتصادي. أما بالنسبة للبلدان النامية فانهم يعانون من نص في العملات الأجنبية التي يمكن استخداما لتمويل الواردات من السلع والخدمات الاستهلاكية والمواد الخام والمكونات والمدخلات الرأسمالية الجديدة. وبالعودة إلى أساس حساب الدخل القوي يككن اشتقاق جفوة الادخار ، فإن (GDP) هو إجلالي الناتج المحلي المنتجة في سنة معينة، (C) هو الاستهلاك الخاص و (I) هو الاستثمار، و (G) هو استهلاك الحكوي، (X) يدل على الصادرات، (M) يمثل الواردات، و (S) هو الادخار، (T) تقف على إجلالي الإيرادات الضريبة الحكومية. إذ إننا نعرف أن (BON:2014,7)

.(Raji:2019,94)

$\mathrm{Y}=\mathrm{C}+\mathrm{I}+\mathrm{G}+\mathrm{X}-\mathrm{M} \ldots \ldots 18$

(C+S+T) يسإعادة ترتيب نحصل على (Y)

$\mathrm{C}+\mathrm{I}+\mathrm{G}+\mathrm{X}-\mathrm{M}=\mathrm{C}+\mathrm{S}+\mathrm{T} \ldots \ldots .19$

لذا فإن

$$
\mathrm{S}-\mathrm{I}=(\mathrm{X}-\mathrm{M})+(\mathrm{G}-\mathrm{T}) \ldots \ldots .20
$$

وهذا يعطينا المعادلة التي تشرح فيها فجوة الموارد الإجالية للاقتصاد في التوازن الداخلي، أي الموازنة العامة والفجوة الخارجية (الميزان التجاري). إذا بقيت جفوة المدخرات - الاستثمار على حالها أو مستقرة، فإن التغيرات في السياسات التي تزيد من جزز الموازنة ستزيد من جزز الحساب التجاري، والعكس
يسبب في زيادة الطلب على السلع المحلي. فضلًا عن ذلك فإنه يشكل الحقن في الاقتصاد على العكس من ذلك، فإن الادخار(S) والضرائب (T)، والواردات يسبب في انخفاض الطلب على السلع المحلية؛ ممائدي إلى التسرب في هذا الاقتصاد. إن النفقات الكلية في السلع في أي اقتصاد قد تعطينا المكافئ في معادلة رقة (10)، الإيرادات المتحصل عليها من هذا العرض في مكافٔ رق (11).

$$
\begin{aligned}
& A E=C+I+G+X \ldots \ldots 10 \\
& Y=C+S+T+M \ldots \ldots .11
\end{aligned}
$$

وبالرجوع إلى صياغة المعادلات الأعلى في إطار فرضية توءَمة العجز نرى بأن صياغة فرضية "ثلاثي العجز" تمتمد منها، وهذا من خلال ترتيب وإعادة المعادلات وإدراج المثغير الآخر خاص بالادخار الاستثاري كما نرى (Raji:2019,94): income $(\mathrm{Y})=\mathrm{C}+\mathrm{S}+\mathrm{T} \ldots \ldots .12$

expenditure $(\mathrm{E})=\mathrm{C}+\mathrm{I}+\mathrm{G}+(\mathrm{X}-\mathrm{M}) \ldots \ldots .13$

$$
\text { والمعادلتان متساويتان }
$$

\section{$C_{+} S_{+} T_{=} \mathrm{C}+\mathrm{I}+\mathrm{G}+\mathrm{X}-M \ldots \ldots 14$}

والمعادلتان (10) و(11) متساويتان مع بعضها البعض لأن النفقات يجب أن تكون قادرة على إنتاج السلع الكثيرة لضمان التوازن في الاقتصاد، وبهذا نحصل على المعادلة رق (15).

\section{$C_{+} S_{+} T_{+} M=\mathrm{C}+\mathrm{I}+\mathrm{G}+\mathrm{X} \ldots \ldots 15$}

حصلنا على المعادلة رقٌ (16) من خلال إعادة الترتيب للمعادلة رخٌ (15)، إذ تشير المعادلة إلى أن الحقن يساوي مع التسريبات. $\mathrm{S}+\mathrm{T}+\mathrm{M}=\mathrm{I}+\mathrm{G}+\mathrm{X} \ldots \ldots .16$

والمعادلة رق (17) تشير إلى مصدر العجز الداخلي في الاقتصاد، وقد حصلنا عليه من خلال إعادة وترتيب معادلة رقٌ (16).

$$
(\mathrm{S}-\mathrm{I})+(\mathrm{T}-\mathrm{G})=(\mathrm{X}-\mathrm{M}) \ldots \ldots 17
$$

تعبر معادلة رقة (17) التوازن الداخلي والخارجي للاقتصاد. في الجانب الأيسر (S-I) هو التوازن القطاع الخاص (الادخار - الاستثمر) و (T-G) يشير إلى التوازن في القطاع العام وهو الموازنة العامة. و X-M) في الجانب الأيمن يعرض التوازن الخارجي. وتبين هذه المعادلة أنه إذا كان لدى الاقتصاد العجز الخارجي، يككن أن يكون راجعًا إلى الفجوة الادخارية، أي (الادخار - الاستثمار) في القطاع الخاص و / أو عزز الموازنة العامة. وإذا كان التوازن الخارجي مصحوبًا بواقع 


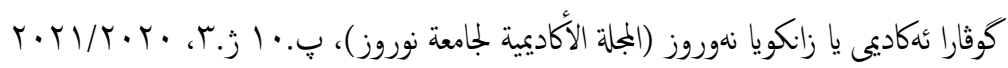

المشاهدات من ظواهر متعددة التي حُصل عليها في فترات زمنية لنفس

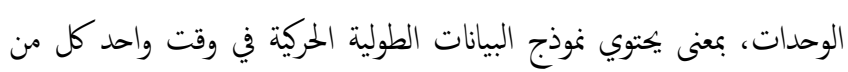
البيانات المقطعية وفترات الزمنية.

تقوم البيانات اللوحية الديناميكية (dynamic panel model) على أساس نماذج الانحدار الذاتي التي تتميز بإضافة متغير داخلي (متغير تابع) أو أكثر بفترة زمنية متباطئة كتغير خارجي مفسر إلى جانب المتغيرات المارجية الأخرى المفسرة

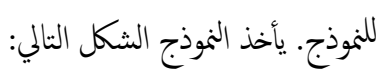

$$
\begin{aligned}
\mathrm{CA}_{\mathrm{it}}=\delta \mathrm{CA}_{\mathrm{it}-1}+\beta_{1} \mathrm{~PB}_{\mathrm{it}}+\beta_{2} \mathrm{SAV}_{\mathrm{it}}+\beta_{2} \mathrm{TRADE}_{\mathrm{it}} \\
+\beta_{2} \mathrm{EXC}_{\mathrm{it}}+\mathrm{U}_{\mathrm{i}}+\mathrm{V}_{\mathrm{it}} \ldots \ldots .21
\end{aligned}
$$

حيث إن (CA) هو يجز رصيد الحساب الجاري، وهو المتغير الحركي ويعتمد

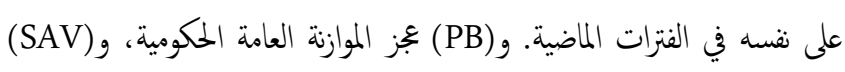

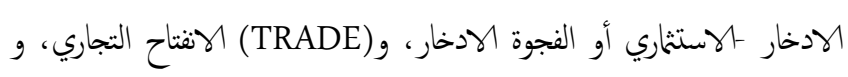
أسعار الصرف. وقد أخذت البيانات عن كل المتغيرات بالنسبة إلى (EXC) الناتج المحلي الإجالي لتفادي تشويه البيانات ونتابُج التحليل. ويعبر كل من t=1,2,.....T. , الوحدة رقة (i) في البيان المقطعي، ويرمز إلى الفترة الزمنية رقٌ (t). ومن المفترض أن أكبر عدد وحدات أو مشاهدات المقطعية هو (N) وأكبر عدد من الفترات الزمنية هو (T)، إذا كانت كل وحدة مقطعية أي دولة لها نفس العدد من مشاهدات السلاسل الزمنية تسمى البيانات الطولية بالبيانات، ويختلف هذا النوع من البيانات عن النوعين الأول والثاني من حيث أنه غني بالمعلومات ويوفر

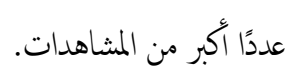
نظرًا لعدم إمكنية قياس وتقدير تلك النماذج التي تحتوي على هذا النوع من البيانات معًا في نموذج واحد عن طريق بيانات السلاسل الزمنية فقط أو البيانات المقطعية فقط. وذلك بسبب عدم دقة التقديرات من بهة وتحتاج أيضًا إلى إجراء انحدارات كثيرة لكل النموذج؛ لذا من الممكن اللجوء إلى قياس العلاقات بين تلك المتغيرات من خلال استخدام البيانات الطولية الديناميكية أو حتى البيانات الطولية العادية (panel data)، ضمن النموذج الواحد (-Gujarati:2003,638

ومن الضروري في البداية معرفة الارتباط بين المتغيرات من خلال تقدير مصفوفة

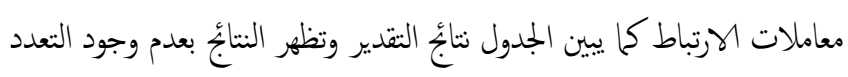

بالعكس، فإن زيادة العجز النجاري ستزيد من عجز الموازنة.

3. المبحث الثاني

1.3

في هذه الدراسة استخدمت البيانات السنوية عن العجز المالي والعجز في الحساب الجاري والفجوة الادخارية والافتّاح النجاري وأسعار الصرف. وتتضمن العينة من (21) من البلدان المتقدمة في منظمة النعاون الاقتصادي، وقد أخذت البيانات عن المتغيرات من (قواعد بيانات منظمة التعاون والتنمية في الميدان Organization for Economic Co-) (OECD) 19تصادي)، operation and Development الاقتصادية وإلى إنعاش التبادلات التجارية. تتكون المنظمة من مجموعة من البلدان المتقدمة التي تقبل مبادئ الديمقراطية التمثيلية واقتصاد السوق الحر. أنشأت منظمة التعاون الاقتصادي والتنمية في 30 من سبتمبر سنة 1961 بعد أن حلت محل منظمة النعاون الاقتصادي الأوروبي OEEC التي أسست سنة 1948. وبعد فترة وسّعت لتشمل عضويتها بلدان غير أوروبية. هذه البلدان شأنها شأن البلدان الأخرى في العالم، بدأ في الماضي سياساتهم الموبجة نخو السوق مثل تحرير الصناعة والتجارة وأسعار الصرف. وكان الغرض من اتباع هذه السياسة هي تشجيع الصادرات وتحتيق المو السيع للاقتصاد. إن المدة الزمنية للعينة المختارة للدراسة متشاهة تمامًا لكل البلدان. وحُصل على هذه البيانات من خلال مصادر موثوقة. وعلى الرغز من أن التركيز الرئيس لهذه الدراسة هو على العلاقة بين المتغرات الأساسية التي تتعلق بتوعَمة العجز وثلاثية العجز، إلا أنها تحاول تسليط الضوء على متغيرات أخرى في الدراسة وتأثيرها على الاقتصاد. تحاول الدراسة اختبار فرضية "العجز الثلاثي" من خلال تحتيق الطرق الإحصائية وتحليل البيات اللوحية الديناميكية (dynamic panel model) لـ (21) دولة ذات اقتصادات المتحولة للمدة (2009-2017). وقد استُخدمت قاعدة البيانات المدجة من المقاطع والمدة الزمنية. وبذلك يكون عدد المشاهدات (189) مشاهدة، وهذه الطريقة أي البيانات اللوحية الديناميكية (dynamic panel model) تستخدم عندما يكون لدينا دول متعددة وسلسلة زمنية أو ما يسمى بـ "المقطعية multiple ) الزمنية"، أو عندما يكون نوع البيانات متعددة الأبعاد dimension) 


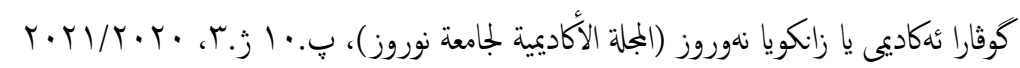

ومن مزايا الطريقة: - (رضا وفريد :2020, 205) (Wooldridge:2001,97):

الخطي بين المتغيرات المستقلة.

تعمل هذه الطريقة على إزالة الارتباط التسلسلي أو الارتباط الذاتي.

$$
\text { تعمل طريقة العزوم المعمّمة على إزالة اختلاف التباين }
$$

تعمل على تصحيح المتغبر النابع (Endogeneity) عن طريق تحويل المتغيرات المستقلة من خلال أخذ الفرق.

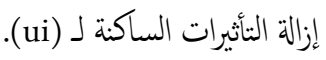

استخدام أدوات المتغيرات (instrument variable). ويستخدم المتغيرات الخارجية كأدوات في هذا النوع من التقدير. ويلاحظ هنا بأنه يككن استخدام الأدوات الداخلية إلا أنه غير مشاهد ولا يككن مشاهدة بياناته. بعكس المتغيرات الخارجية فيمكن مشاهدة بياناتها. الحصول على مقدرات متسقة وغير متحيزة.

ومن مزايا استخدام أسلوب البيانات اللوحية الديناميكية ( dynamic (panel models: GMM ( مع المتغيرات المستقلة، بل فقط يرتبط مع الحد الخطأ ( $\left.\mathrm{U}_{i}\right)$

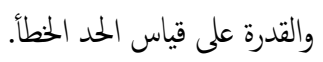

من أجل حساب المتغر التابع المتأخر ( endogeneity ydepentent variable) العزوم المعمّمة ) Moment ولمصصول على مقدرات أكثر كفاءة من المربعات الصغرى ذات المرحلتين أو المربعات الصغرى الوعتيادية، يكننا إضافة شروط اللحظية بافتراض أن القيم السابقة للمتغيرات التفسيرية، أو حتى القيم السابقة للمتغير التابع، غير مرتبطة بصطلح الخطأ (ui)، على الرغ户 من أنها لا تظهر في النموذج.

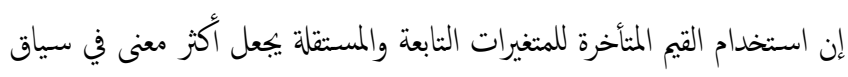
الناذج المقدرة تحت التوقعات العقلانية. ثم إن مصطلح الخطأ في المعادلة غير مرتبط بجميع المتغيرات للفترات المؤخرة في وقت سابق. ويكن أن نصل إلى نتائج التقدير من خلال اختبارين: الأول خاص للتحديد المفرط للقيود (Sargan test of over-identifying restrictions)، والذي يختبر
الجدول (1): مصفوفة ارتباط معاملات

Covariance Analysis: Ordinary

Date: 03/07/20 Time: 07:17

Sample: 20092017

Included observations: 189

\begin{tabular}{|c|c|c|c|c|}
\hline \multicolumn{5}{|c|}{ Correlation } \\
\hline \multicolumn{5}{|c|}{$\mathrm{t}$-Statistic } \\
\hline \multicolumn{2}{|c|}{ ProbabilityPB } & SAV & TRADE & EXC \\
\hline$\overline{\mathrm{PB}}$ & 1.000000 & & & \\
\hline \multirow[t]{3}{*}{$\overline{\mathrm{SAV}}$} & -0.092735 & 1.000000 & & \\
\hline & -1.273627 & 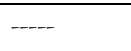 & & \\
\hline & 0.2044 & - & & \\
\hline \multirow[t]{3}{*}{ TRADE } & 0.142809 & 0.005066 & 1.000000 & \\
\hline & 1.973113 & 0.069278 & $\ldots$ & \\
\hline & 0.0500 & 0.9448 & $\ldots$ & \\
\hline \multirow[t]{3}{*}{ EXC } & 0.031463 & -0.086341 & -0.061072 & 1.000000 \\
\hline & 0.430460 & -1.185126 & -0.836708 & -- \\
\hline & 0.6674 & 0.2375 & 0.4038 & $\cdots$ \\
\hline
\end{tabular}

dynamic panel ( 2.1.3 تقدير وتحليل نموذج البيانات اللوحية الديناميكية

(data model

في هذه الدراسة نطبق طريقة البيانات اللوحية الديناميكية لثقنية العزوم المعمّمة dynamic panel model Generalised Method of Moment ) GMM )، لحساب المقطعية الزمنية. إن تقدير البيانات الطولية بهذه الطريقة يعتمد Lee \& Wang ) على مجموعة من الشروط والافتراضات، من بينها (Wooldridge:2001,97-) (Baltagi:2005,136-137) (:2015,705

$$
\text { يعتمد المتغير التابع على نسه في الماضي. }
$$

not strictly ) إن المتغيرات المستقلة ليست خارجية بكتة exogenous ). ولها ارتباط مع الخطأ في الماضي أو يمكن في الحالي. تتمتع بالكفاءة العالية خاصة عندما تكون الفتزة الزمنية أقل والمقطع العرضي أكبر T<N، أي يجب أن يكون عدد الوحدات أكبر من الفترة الزمنية. ومن ناحية أخرى فإن زيادة ججم العينة يؤدي إلى زيادة الدرجات الحرية بالتالي إلى كفاءة الموذج المقدر. يجب أن يكون عدد أدوات المتغيرات (instrument variable) من المتغيرات الداخلية (endogenous). 
difference Generalised الفرق العزيقة المعمة - 1.3.1.3

Method of Moment- GMM

وقد طورت هذه الطريقة من قبل (Arellano \& Bond 1991) وتعمل

هذه الطريقة على تصحيح النداخل عن طريق تويل المتغيرات المستقلة من خلال

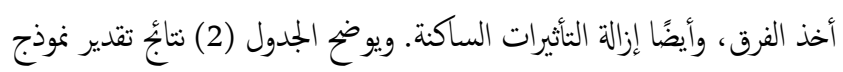
مقدّر GMM للفرق من خطوة والخطوتين. ونلاحظ من خلال الجدول بأن كل من العجز الملالي والفجوة الادخارية والمتغير التابع المتخلف زمنئًا لها تأثيرات معنوية على عزز الحساب الجاري، وكانت الثاثثيرات ذات إثارة موجبة، أي وجود علاقة طردية بين كل من العجز المالي والفجوة الادخارية. وبناءً على ذلك، فإن زيادة العجز المالي والفجوة الادخارية حتى جز الحساب الجاري على نفه تؤدي إلى زيادة العجز في الحساب. ومن أجل تعزيز وتحسين الحساب الجاري يجب اتباع السياسة المالية الفعالة من خلال إعادة التوازن إلى الموازنة العامة هذا من بجة، ومن بجةٍة أخرى يمكن أن يكون تحسين الحساب الجاري من خلال تشجيع الادخار وبالتالي تعزيز القدرة الاستثمارية وبالتالي يؤدي إلى زيادة أو تحسين

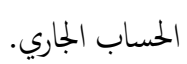

Difference - Generalised Method of Moment الجدول 2: نتاجُ تقدير طريقة

\section{(DF-GMM)}

\begin{tabular}{|c|c|c|c|}
\hline variables & LSDV & $\begin{array}{l}\text { One step FR- } \\
\text { GMM Model } \\
(1 \mathrm{lag})\end{array}$ & $\begin{array}{l}\text { Two step FR- } \\
\text { GMM Model (1 } \\
\text { lag) }\end{array}$ \\
\hline \multirow{2}{*}{ CA $(-1)$} & 0.72964 & 0.478253 & 0.400048 \\
\hline & 0.0000 & 0.0004 & 0.0001 \\
\hline \multirow{2}{*}{$\mathrm{PB}$} & 0.15963 & 0.510382 & 0.497648 \\
\hline & 0.0032 & 0.0000 & 0.0018 \\
\hline \multirow{2}{*}{$\mathrm{PB}(-1)$} & -0.1307 & -0.0057 & 0.045012 \\
\hline & 0.0033 & 0.9458 & 0.7861 \\
\hline \multirow{2}{*}{ SAV } & 0.08807 & 0.072941 & 0.083603 \\
\hline & 0.0000 & 0.0016 & 0.0004 \\
\hline \multirow{2}{*}{$\operatorname{SAV}(-1)$} & 0.00239 & 0.015656 & -0.003218 \\
\hline & 0.8657 & 0.6538 & 0.9181 \\
\hline \multirow{2}{*}{ F-STATISTIC } & 187.962 & & \\
\hline & 0.0000 & & \\
\hline SARGAN & & 0.0188 & 0.287 \\
\hline $\begin{array}{l}\text { Arellano-Bond } \\
\text { AR (2) }\end{array}$ & & 0.308 & 0.966 \\
\hline $\mathrm{n}$ & 168 & 147 & 147 \\
\hline $\begin{array}{l}\text { Instrument } \\
\text { rank }\end{array}$ & & 38 & 21 \\
\hline
\end{tabular}

Eviews-10 المصدر: من عمل الباحثين بالاعتاد على نتأُج برنامج
جودة الأدوات المستعملة في النموذج، بجيث تتمثل الفرضية (H0). وينص الاختبار على صلاحية الأدوات عند قبول فرضية العدم. والاختبار الثاني خاص بالارتباط التسلسلي أو الارتباط الذاتي من (Arellano and Bond) الدرجة الثانية (2) AR والذي يفحص فرضية أن حد الخطأ لا يرتبط تسلسليًا، حيث نختبرها ما إذا كان الثفاضل حد الخطأ يرتبط تسلسيليًا من الدرجة الثانية (p-value>0.05) وهو قبول فرضية العدم (H1) إذ (second-order) إن عدم رفض الفرضيات العدمية لكلا الاختبارين يعطي دعمًا للموذج. والفشل عند رفض فرضية العدم عندما يكون ليس هناك فرق الثاني للارتباط التسلسلي .(Arelino:1991,278-) (Baltagi:2005,136-137)

\section{Generalised Method of نثائج تقدير طريقة العزوم المقمّة 3.1.3}

في ضوء الافتراضات والشروط السابقة المشار إلها أعلاه سيقدر نموذج Generalised ( باستخدام تقنية العزوم المعممة (dynamic panel model) Method of Moment GMM لتقدير الناذج التي تتتوي البيانات الطولية مع المتغيرات التفسيرية الذاتية التي لا تظهر بشكل مضاف في المعادلة. وتوضح الخصائص الديناميكية أن الثاثثرات الثابتة الخاصة بكل بلد يكن أن يرتبط بمتغير تابع ختتلف، وأن بعض المتغيرات التفسيرية قد تكون داخلية المنشأ، ويككن أن يجعل حالة عدم التناسق من خلال طريق المربعات الصغرى الاعتيادية (OLS) وتكون النتائُ تقديرات متهيزة (Wooldridge:2001,90-93) الحتيقي لطريقة العزوم المعمّمة تكون كالتالي: -

$$
\begin{aligned}
& \mathrm{CA}_{\mathrm{it}}= \delta \mathrm{CA}_{\mathrm{it}-1}+\beta_{1} \mathrm{~PB}_{\mathrm{it}}+\beta_{2} \mathrm{SAV}_{\mathrm{it}}+ \\
& \beta_{2} \mathrm{TRO}_{\mathrm{it}}++\beta_{2} \mathrm{EXC}_{\mathrm{it}}+\mathrm{U}_{\mathrm{i}}+\mathrm{V}_{\mathrm{it}} \ldots \ldots .22 \\
&- \text { والموذج المول }
\end{aligned}
$$

$\Delta \mathrm{CA}_{\mathrm{it}}=\Delta \delta \mathrm{CA}_{\mathrm{it}-1}+\Delta \beta_{1} \mathrm{~PB}_{\mathrm{it}}+\Delta \beta_{2} \mathrm{SAV}_{\mathrm{it}}+$

$$
\Delta \beta_{2} \mathrm{TRO}_{\mathrm{it}}+\Delta \beta_{2} \mathrm{EXC}_{\mathrm{it}}+\Delta \mathrm{v}_{\mathrm{it}} \ldots \ldots .23
$$

وبتحويل النموذج من خلال أخذ الفرق للمتغيرات المستقلة، أُزيلت تأثيرات

$$
\text { السكون المتمثلة بـ ( } \left.)_{i}-\mathrm{U}_{\mathrm{it}-1}\right) .
$$

Panagiotis \& ) - وتنقسم طريقة العزوم المعمّمة إلى نوعين من التقديرات وهات - (Michael: 2018,12 


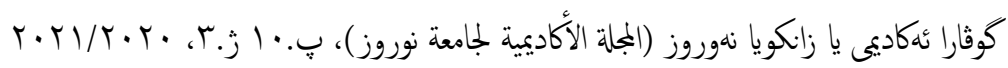

SYSTEM - Generalised Method of Moment الجدول 3. نتاجُ تقدير طريق (SYS-GMM)

\begin{tabular}{|c|c|c|}
\hline variables & $\begin{array}{l}\text { One step SYS- } \\
\text { GMM Model (1 } \\
\text { lag) }\end{array}$ & $\begin{array}{l}\text { Two step SYS- } \\
\text { GMM Model (1 } \\
\text { lag) }\end{array}$ \\
\hline \multirow{2}{*}{ CA $(-1)$} & 0.53539 & 0.47711 \\
\hline & 0.0000 & 0.0000 \\
\hline \multirow{2}{*}{$\mathrm{PB}$} & 0.45930 & 0.44070 \\
\hline & 0.0000 & 0.0260 \\
\hline \multirow{2}{*}{$\mathrm{PB}(-1)$} & -0.05520 & 0.01458 \\
\hline & 0.3254 & 0.9371 \\
\hline \multirow{2}{*}{ SAV } & 0.05093 & 0.07568 \\
\hline & 0.1158 & 0.2329 \\
\hline \multirow{2}{*}{$\operatorname{SAV}(-1)$} & 0.00478 & -0.02482 \\
\hline & 0.8700 & 0.5230 \\
\hline SARGAN & 0.100 & 0.264 \\
\hline Arellano-Bond AR (2) & & 0.44 \\
\hline $\mathrm{n}$ & 147 & 147 \\
\hline Instrument rank & 38 & 21 \\
\hline
\end{tabular}

الادخارية. وعند قراءة الدلالات الإحصائية والمعايير الخاصة بتقييم النموذج نرى بأن هناك تقاربًا في النتأُج مع طريقة الفرق في بعض المعايير؛ لذا يجب أن نلجأ إلى المقارنة بين النموذجين بهدف اختيار النموذج الملائي. وبالنسبة للجزء السفلي للجدول نرى بأن الإشارة والدلالات تشير إلى إمكنية قبول فرضية العدم الخاص

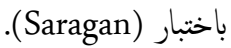

4.1.3 اختيار بين طريقة الفرق والنظام

في هذا الجزء تتم المقارنة بين النموذجين واختيار الأفضل فيا بينها من خلال

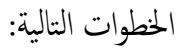

إجراء وتقدير طريقة نموذج الانحدار التجميعي، (POLS) تفترض هذه الطريقة على أنه ليس هناك أي تمايز بين الوحدات ولا في الزمن. وهذا الافتراض يعدّ بأن كل المعلمات تتمتع بالتجانس الكمل. نموذج الثأثيرات الثابتة، تقتح هذه الطريقة بوجود تأثيرات فردية لكل دولة، بحيث معلمات الميل هي ثابتة عبر الوحدات وعبر الزمن ببنا الثابت يختلف رت

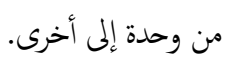

وفي الخطوة الأخيرة نأتي إلى إجراء المقارنة بين النموذجين من خلال مقارنة معلمة المقدرة للمتغير التابع المتأخر، ثم اختيار النموذج الأمثل. إذا كانت معلمة نموذج الفرق المقدر (DF-GMM) قريبًا أو أقل من المعلمة المقدرة
ويعرض الجزء السفلي من الجدول ملخصًا إحصائيًا من الخزجات حول تلك

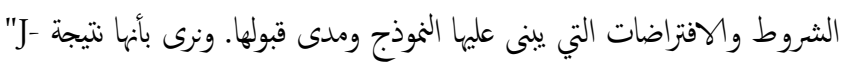
"statistic و "ترتيب الأداة" (instrument) ورتبة أداة 21 وهي أكبر من عدد المعاملات المقدرة في النموذج الخطوتين (2) AR وهو قبول فرضية العدم وقد نستخدها لإنشاء اختبار سارجان (sargan) للقيود المفرطة في تحديد صلاحية الأدوات المستعملة. وتجدر الإثشارة هنا إلى أن الإحصاء (J-statistic) الموضح في المعادلة اللوحية هي (Prob (J statistic=0.28). وهو رفض فرضية العدم ( $\left.1 \mathrm{i}_{0}\right)$ أما فيا يتعلق باختبار (Arellano-Bond) اختلفت ننائُ الفرق في تقدير GMM Arellano-Bond أن احتالية إحصائية لهذا الوختبار أكبر من (0.05\%) أي قبول الفرضية العدمية، والتي تنص على عدم وجود الارتباط التسلسلي أو الارتباط الناتي من الدرجة الثانية لحد الخطأ العشوائي، وهذا ما يدل على صلاحية قيود العزوم المستعملة في التقدير.

\section{System Generalised Method of نظام 2.3.1.3 طريقة العزوم المعممة - نظمة \\ Moment- GMM}

وقد طبقت هذه الطريقة (SYS-GMM) من قبل ( Arellano \& Bover) 1995) و (Blundell \& Bond 1998) وقد جرت آلية عمل هذه الطريقة على تصحيح المتغير التابع المتأخر من خلال إدخال وتعريف كثير من الأدوات لتطوير سرعة وتحسين كفاءة النموذج لفصل المتغيرات الخارجية مع التأثيرات الساكنة وبناء نظام للمعادلتين: المعادلة الحقيقية أو الأصلية والمعادلة المحولة، كتالي: System- Generalised ) النموذج الحتيقي لطريقة العزوم المعمّمة نظام كالتالي: - Method of Moment System- Generalised Method of ( في طريقة العزوم الممثّعة- النظام (Moment الفرق والأدوات (instrument variable) وتعبر المعادلة الثانية عن شكل

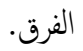

كما يوضح الجدول SY) نتائج تقدير نموذج مقدّرSYS- GMM من خطوة والخطوتين. نرى بأن هناك تأثيرًا للمتغيرات على العجز الحساب، وكانت الثأثيرات ذات الإثارة الموجبة، أي وجود علاقة طردية بين كل من العجز المالم والفجوة 


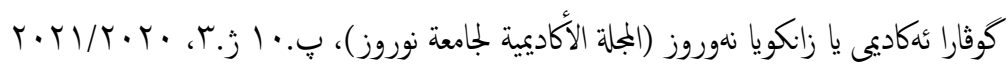

الجدول 5. نتاجُ بيانات جذر الوحدة عند المستوى الأصلي

\begin{tabular}{|c|c|c|c|c|c|c|}
\hline \multicolumn{7}{|c|}{ 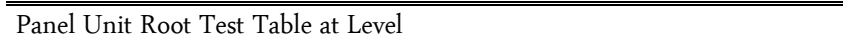 } \\
\hline \multicolumn{7}{|c|}{ Levin, Lin \& Chu } \\
\hline & & CA & PB & SAV & TRADE & EXC \\
\hline \multirow[t]{2}{*}{ intersept } & $\mathrm{t}$-Statistic & -5.0648 & -9.456 & -11.540 & -6.2637 & -0.1163 \\
\hline & $\begin{array}{l}\text { Prob. } \\
\text { P }\end{array}$ & 0.0000 & 0.000 & 0.0000 & 0.0000 & 0.4537 \\
\hline intercept & $\mathrm{t}$-Statistic & -9.1475 & -11.701 & -11.468 & -11.462 & -8.5859 \\
\hline \& Trend & Prob. & 0.000 & 0.0000 & 0.0000 & 0.0000 & 0.0000 \\
\hline \multicolumn{7}{|c|}{ Im, Pesaran and Shin W-stat } \\
\hline \multirow[t]{2}{*}{ intersept } & $\mathrm{t}$-Statistic & -2.2011 & -0.0171 & -6.427 & -1.9003 & 2.25762 \\
\hline & $\begin{array}{l}\text { Prob. } \\
\text {. }\end{array}$ & 0.0139 & 0.4932 & 0.0000 & 0.0287 & 0.9880 \\
\hline intercept & $\mathrm{t}$-Statistic & -0.8213 & -1.261 & -2.222 & -1.171 & -1.5579 \\
\hline$\&$ Trend & Prob. & 0.2057 & 0.103 & 0.0131 & 0.0000 & 0.0597 \\
\hline \multicolumn{7}{|l|}{$\mathrm{ADF}$} \\
\hline \multirow[t]{2}{*}{ intersept } & $\mathrm{t}$-Statistic & 73.380 & 52.56 & 126.77 & 65.5383 & 28.5710 \\
\hline & $\begin{array}{l}\text { Prob. } \\
\text {. }\end{array}$ & 0.0019 & 0.127 & 0.0000 & 0.0115 & 0.9112 \\
\hline intersept & $\mathrm{t}$-Statistic & 61.934 & 70.440 & 99.905 & 68.7880 & 79.439 \\
\hline \& Trend & Prob. & 0.0242 & 0.0039 & 0.0000 & 0.0057 & 0.0002 \\
\hline \multicolumn{7}{|l|}{ PP } \\
\hline \multirow[t]{2}{*}{ intersept } & $\mathrm{t}$-Statistic & 74.001 & 38.20 & 163.372 & 46.7453 & 36.3736 \\
\hline & Prob. & 0.0017 & 0.638 & 0.0000 & 0.28838 & 0.6343 \\
\hline intersept & $\mathrm{t}$-Statistic & 66.423 & 91.057 & 158.538 & 122.938 & 121.669 \\
\hline \& Trend & $\begin{array}{l}\text { Prob. } \\
\text {. }\end{array}$ & 0.0096 & 0.000 & 0.0000 & 0.0000 & 0.0000 \\
\hline
\end{tabular}

وكذلك يتينن من الجدول (6) آن جميع المتغيرات ساكنة بعد أخذ الفرق. كما تظهر غالبية نتاجُج الاختبارات، سكون البيانات اللوحية؛ مما يعني إمكانية إجراء التكامل

المشترك للبيانات.

\section{الجدول 6. نتاجُ بياتات جذر الوحدة عند الفرق الأول}

\begin{tabular}{|c|c|c|c|c|c|c|}
\hline \multicolumn{7}{|c|}{ Panel Unit Root Test Table at the firs difference } \\
\hline \multicolumn{7}{|c|}{ Levin, Lin \& Chu } \\
\hline & & CA & PB & SAV & TRADE & EXC \\
\hline \multirow[t]{2}{*}{ intersept } & t-Statistic & -11.792 & -14.776 & -17.057 & -7.5070 & -11.547 \\
\hline & $\begin{array}{l}\text { Prob. } \\
\text { P }\end{array}$ & 0.0000 & 0.0000 & 0.0000 & 0.0000 & 0.0000 \\
\hline intersept & t-Statistic & -9.9962 & -20.107 & -20.859 & -2.3786 & -18.425 \\
\hline$\&$ Trend & 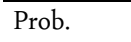 & 0.000 & 0.0000 & 0.0000 & 0.0087 & 0.0000 \\
\hline \multicolumn{7}{|c|}{$\mathrm{Im}$, Pesaran and Shin W-stat } \\
\hline \multirow[t]{2}{*}{ intersept } & t-Statistic & -5.1017 & -6.9165 & -8.7079 & -2.3701 & -6.9465 \\
\hline & $\begin{array}{l}\text { Prob. } \\
\text { P }\end{array}$ & 0.0000 & 0.0000 & 0.0000 & 0.0089 & 0.0000 \\
\hline intersept & t-Statistic & -0.4592 & -2.7621 & -2.1999 & 1.7704 & -2.255 \\
\hline \& Trend & Prob. & 0.0000 & 0.0000 & 0.0000 & 0.0962 & 0.0120 \\
\hline \multicolumn{7}{|l|}{$\mathrm{ADF}$} \\
\hline \multirow[t]{2}{*}{ intersept } & $\mathrm{t}$-Statistic & 104.555 & 135.510 & 157.486 & 68.1838 & 131905 \\
\hline & Prob. & 0.0000 & 0.0000 & 0.0000 & 0.0065 & 0.0000 \\
\hline intersept & $\mathrm{t}$-Statistic & 54.3737 & 105.069 & 91.1343 & 17.0794 & 95.967 \\
\hline \& Trend & Prob. & 0.0955 & 0.0000 & 0.0000 & 0.0964 & 0.0000 \\
\hline \multicolumn{7}{|l|}{$\mathrm{PP}$} \\
\hline \multirow[t]{2}{*}{ intersept } & $\mathrm{t}$-Statistic & 124.200 & 172.861 & 196.793 & 68.2428 & 212.665 \\
\hline & Prob. & 0.0000 & 0.0000 & 0.0000 & 0.0998 & 0.0000 \\
\hline intersept & t-Statistic & 109.302 & 180.138 & 148.055 & 25.141 & 179.324 \\
\hline$\&$ Trend & Prob. & 0.0000 & 0.0000 & 0.0000 & 0.09817 & 0.0000 \\
\hline
\end{tabular}

لنموذج الثأثيرات الثابتة للمتغير التابع المتأخر والذي يدل على تقدير متحيز،

ففي هذه الحالة من الأفضل أن نختار النموذج العزوم المعمّمة- النظام

$$
\text { (SYS-GMM) }
$$

الجدول 4. نتاجُ تقدير قمة معلمة المقدرة للمتغر التابع المتأخر في النموذجين الثاثيرات الثابتة وتثنية العزوم المعقعة ذات الفرق المقاع

\begin{tabular}{lll}
\hline \hline & Fixed effects Model & $\begin{array}{l}\text { One \& Two steps } \\
\text { Differences GMM }\end{array}$ \\
\hline CA (-1) & 0.72964 & 0.478253 \\
& & 0.40004 \\
\hline \hline
\end{tabular}

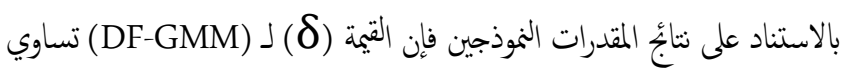
(0.400) وهي أقل من قيمة معلمة نموذج المقدر الخاص بالثأثيرات الثابتة، وهي تبلغ (0.72)، وهذا يفرض على أفضلية استخدام طريقة العزوم المعمّمة - النظام وهي أكثر (System Generalised Method of Moment- GMM) ملاءمة من طريقة العزوم المعمّمة - الفرق ( Differences Generalised .(Method of Moment- GMM

\section{Pooled Mean Group تقدير البيانات اللوحية من خلال طريقة} PMG and Mean Group MG Estimation هناك طريقة أخرى يككن أن نستعين بها لمعرفة وجود العلاقة بين المتغيرات النموذج في الأمدين الطويل والقصير، وتسعى الدراسة في هذا الجزء إلى الوصول إلى تصحيح الأخطاء عن طريق تقدير النموذج PMG للتحقق من وجود العلاقة بين متغيرات النموذج. وقبل القيام بذلك، يجب إجراء اختبارات السكون واختبار التكامل المشترك. لأهها تتطلب طريقة أو نموذج تصحيح الأخطاء المستند إلى تقدير PMG وجود تكمل مشترك بين المتغير التابع والمتغيرات التوضيحية. لذلك، تختبر الدراسة أولاً سكون المتغيرات باستخدام مجموعة من الاختبارات. 1.5.1.3 نتاجُ اختبارات السكون قبل بدء بناء الهيكل للبيانات أو النموذج يجب أن نتأكد من خلو البيانات اللوحية من جذر الوحدة، ولدينا اختبارات مختلفة لهذا الغرض مثل ( Levin, Lin \& (PP) , (ADF) , Im, Pesaran and Shin W-stat)) , Chu) وحسب ننائُ الاختبارات في الجدول رةٌ (5) نرى بأن البيانات اللوحية غير ساكنة في المستوى، عدا المتغير الثالث وهو الفجوة الادخارية تكون ساكنة في المستوى الأصلي، بمعنى أن البيانات تحتوي على جذر الوحدة للمتغيرات عند المستوى.كما نرى في الجدول نتيجة السكون في المستوى. 
Estimation الدراسة، ثم نكشف نتابُج من خلال بمجوعة من الاختبارات والمنوذج الذي يُقدّر

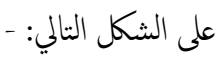

\section{الجدول 8. نتاجُ اختبار (kao) للتكمل المشترك للبيات اللوحية الديناميكية}

\section{Kao Residual Cointegration Test}

Series: CA EXC PB SAV TRADE

Date: 03/10/20 Time: 06:55

Sample: 20092017

Included observations: 189

Null Hypothesis: No cointegration

Trend assumption: No deterministic trend

Automatic lag length selection based on SIC with a max lag of 1

Newey-West automatic bandwidth selection and Bartlett kernel

\begin{tabular}{lll}
\hline ADF & t-Statistic & Prob. \\
\cline { 2 - 2 } & -4.847569 & 0.0000 \\
\hline Residual variance & 2.094832 & \\
\hline HAC variance & 1.971205 &
\end{tabular}

Augmented Dickey-Fuller Test Equation

Dependent Variable: D(RESID)

Method: Least Squares

Date: 03/10/20 Time: 06:55

Sample (adjusted): 20112017

Included observations: 147 after adjustments

\begin{tabular}{lllll}
\hline Variable & Coefficient & Std. Error & t-Statistic & Prob. \\
\hline RESID(-1) & -0.649127 & 0.072781 & -8.918874 & 0.0000 \\
\hline D (RESID (-1)) & 0.207461 & 0.072943 & 2.844156 & 0.0051 \\
\hline R-squared & 0.355509 & Mean dependent var & -0.024447 \\
\hline Adjusted R-squared & 0.351064 & S.D. dependent var & 1.689485 \\
\hline S.E. of regression & 1.360991 & Akaike info criterion & 3.467816 \\
\hline Sum squared resid & 268.5832 & Schwarz criterion & 3.508502 \\
\hline Log likelihood & -252.8845 & Hannan-Quinn criter. & 3.484347 \\
\hline Durbin-Watson stat & 2.138573 & &
\end{tabular}

Eviews-10 المصدر: من عمل الباحثين بالاعتاد على ننائج برنامج

$$
d\left(C A_{t}\right)=\beta+\delta C A_{t-1}+\beta_{1} P B_{t-1}+\beta_{2} S A V_{t-1}
$$

$$
+\beta_{3} \operatorname{TRADE}_{\mathrm{t}-1}+\sum_{\mathrm{i}=1}^{\mathrm{m}} \mathrm{a}_{1, \mathrm{i}}{ }^{*} \mathrm{~d}\left(\mathrm{CA}_{\mathrm{t}-\mathrm{i}}\right)+\sum_{\mathrm{i}=1}^{\mathrm{k}} \mathrm{a}_{2, \mathrm{i}}{ }^{*} \mathrm{~d}\left(\mathrm{~PB}_{\mathrm{t}-\mathrm{i}}\right)
$$

$$
+\sum_{i=1}^{k} a_{3, i}{ }^{*} d\left(\operatorname{SAV}_{t-i}\right)+\sum_{i=1}^{k} a_{4, i}{ }^{*} d\left(\operatorname{TREAD}_{t-i}\right)+u_{i t} \ldots \ldots \ldots . .24
$$

والنموذج الذي نخن بصدد تقديره يتضمن المتغير التابع والمتغير التابع المتأخر والمتغيرات المستقلة، ويأخذ الشكل الذي نرى في المعادلة رُّ (24). وهذا النوع من النموذج يجتاج إلى الشروط النالية: أولاً، يجب أن يكون المتغير التابع بالفرق
2.5.1.3 التكامل المشترك للبيانات اللوحية

هناك بمحوعة من الاختبارات التي يمكن أن ستخدما في اختبار النكمل المشترك مثل (Kedroni) و (Kao). وعند تطبيق الاختبار الأول والذي ينكون من سبعة اختبارات والمعروف (بسبعة اختبارات بيدروني) لتكمل البيانات اللوحية. وتُجرى هذه الاختبارات على كل من الحالتين: مع وجود القاطع ومع القاطع والاتجاه. وتقترح غالبية الاختبارات رفض فرضية العدم. والجدولان (7,8) يبينان نتيجة الاختبار ويؤكدان على وجود التكامل المشترك بين المتغيرات وذلك من خلال مقارنة القيمة الاحتالية مع المستوى المعنوي (5\%) ورفض فرضية العدم (Ho)، وبالتالي فإن النتائج تظهر أن العجز الملالي والفجوة الادخارية والتجارة

$$
\text { وسعر الصرف يتكامل مع عزز الحساب الجاري. }
$$

الجدول 7. نتاجُ اختبار (Pedroni) التكامل المشترك للبيات اللوحية الديناميكية

\begin{tabular}{llll}
\hline \hline Dimension & Test statistics & Intercept & Intercept\& trend \\
\hline \multirow{3}{*}{$\begin{array}{l}\text { Within- } \\
\text { dimension }\end{array}$} & Panel v-Statistic & 0.9890 & 0.9704 \\
\cline { 2 - 4 } & Panel rho-Statistic & 0.9995 & 0.9999 \\
\cline { 2 - 4 } & Panel PP-Statistic & 0.0000 & 0.0000 \\
\cline { 2 - 4 } & Panel ADF-Statistic & 0.0000 & 0.0000 \\
\hline \multirow{3}{*}{$\begin{array}{l}\text { Between- } \\
\text { dimension }\end{array}$} & Group rho-Statistic & 1.0000 & 1.0000 \\
\cline { 2 - 4 } & Group PP-Statistic & 0.0000 & 0.0000 \\
\cline { 2 - 4 } & Group ADF- & 0.0016 & 0.0000 \\
\hline \hline
\end{tabular}

وفيا يتعلق بالاختبار (Kao) فإن النتيجة تدل على رفض فرضية العدم وذلك من

خلال مقارنة القيمة الاحتالية مع المستوى المعنوي (5\%)

Pooled Mean PMG) يكون تقدير البيانات اللوحية من خلال طريقة (Group ARDL Estimation الدرجة نفسها. لأها تتميز بعدة مزايا، يكنن استخداما بغض النظر عن درجة تكمالها، سواء من درجة الصفر (I) (I (I) أو من درجة الواحد (1) (I)، ومن المزايا الأخرى أيضًا يكن استخداهما في تقدير نموذج في الأجلين القصير والطويل معًا في الوقت نتسه (الشوربجي: 2009, 156). ويتم في هذه المنهجة دمج نماذج الانحدار الذاتي ونماذج فترات الإبطاء الموزعة في نموذج واحد. ومن خلال هذه المنهجية يككن أن نحد تأثير ونوع العلاقة النكاملية بين المتغير التابع والمتغيرات المستقلة. يككن تقدير المعلمات في المدى القصير والطويل من خلال إجراء هذا الاختبار في Pooled Mean Group PMG and Mean Group MG ) إطار 


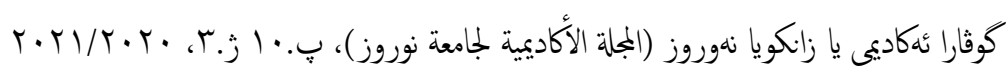

يمتوي النموذجان في الجزء الأول على كل من المتغير التابع المتأخر والمتغيرات المستقلة في الفترات الزمنية الحالية. وهناك تأثير معنوي لكل من (PB) و

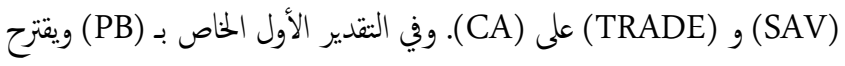
هذا الاختبار بأن معدل الزيادة في العجز المالي بنسبة (1\%) يؤدي إلى زيادة أو تدهور في الحساب الجاري بنسبة (54 \%) و (2.15-\%) كل على التوالي بالنسبة لـ (PMG) و) (MG) أما بالنسبة لمعلمة (SAV) وهي أيضًا إيجابية في النموذج (PMG) والسالبة في النموذج (MG) في المدى الطويل، كما نرى بأن التدهور في الفجوة الادخارية بنسبة (1\%) يؤدي إلى التدهور في الحساب الجاري بنسبة (0.18\%) و (TRADE) (T) وهي أيضًا سلبية في النموذج الأول وإيهابية في الثاني. Pooled PMG) ويتم التعبير عن تقدير نموذج تصحيح الخطأ المستند إلى (Mean Group ARDL Estimation والثالث في المعادلة رق (24)، وفي الحتيقة أن هذه الأجزاء لها أهمية بالنسبة للنموذج، والتي تسمى بـ "المنطقة قصيرة الأجل أو الأجل القصير". ولكي يُصل Error ( على كفاءة النموذج يجب الخصول على قيمة معامل تصحيح الخطأ Correct Term انساقًا كيرًا حول الإثارة والأهمية الإحصائية لهذا التقدير، كما يتين في الجدول (9) أن قيمة معامل تصحيح الخطأ (ECT) التي تعادل (0.51-) و (1.57-) في المنوذجيين الأول والثاني وهي سالبة وقيمته الاحتالية أقل من نسبة (5\%) وهو ما يدع نتائُ الموذج في الأجل الطويل. فالإثارة السالبة تعني أن النموذج يصحح نسه، أي يقضي أو يقلل مستوى ابتعاده عن التوازن، أي يصحح الخطأ بمدل (0.51-) و (1.57-) كل على التوالي سنويًا كما ويدل على سرعة النعديل وقدرته على التكيف نحو الوصول إلى مستوى التوازن الذي يميز علاقة متغيراته فيه بينها

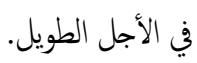
ومن جانب آخر إن آثار العجز الملالي والفجوة الادخارية والتجارة على عز الحساب الجاري في المدى القصير تتعارض مع آثار تلك المتغيرات في المدى الطويل. بالإضافة إلى ذلك، يكون معامل تصحيح الأخطاء لها مواصفات كمة بحيث أنها سالبة ومعنوية بدرجة كيرة عند مستوى (5\%) وهما شرطان أساسيان لوجود علاقة طويلة الأمد، أي بعمنى حتى يكون لدينا تكامل المشترك أو علاقة طويلة الأجل بين المتغيرات المستقلة والمتغير التابع يجب أن تتصف
الأول كما نرى في الجانب الأيسر من المعادلة ـ ثائا، لدينا ثلاثة مجاميع أخرى في المعادلة الجمموعة الأولى المتمثلة بعلومات في الأجل الطويل وهي الجزء الأول في $\left.\left.\beta_{+} \delta \mathrm{CA}_{\mathrm{t}-1}+\beta_{1} \mathrm{~PB}_{\mathrm{t}-1}+\beta_{2} S A V_{t-1}+\beta_{3} \mathrm{TRADE}_{\mathrm{t}-1}\right)\right)$ المعادلة بععى أن البيانات عن المتغيرات في هذا الجزء في المستوى بإبطاء سنة واحدة. والمجموعة الثانية، وهي: إبطاؤه المتغير التابع نفسه، وتبدأ إبطائينه من فترة زمنية واحدة إلى الفترة التي يتطلب فيها النموذج، وهي المتمثلة بالجزء الثاني من يمين المعادلة ( إبطاء المتغيرات المستقلة بالفرق الأول والمتمثلة بالجزء الثالث في المعادلة رخٍ (24)، والتي تبدأ بالإبطاء من فترة صفر إلى الفترة التي ستستخدم في عملية التقدير، ثم يضاف إلى كل ذلك الحد الحطأ المتمثل بـ (ut ). ومن الجدير بالذكر هنا إلى أن

هذا النموذج (ARDL) هو النموذج نفسه الذي طوره (Peasaran -1995). ونستعرض نتانجُ هذا التقدير من خلال الجدول رقّ (9) خاص بنتائُ تقدير النموذج. كما تظهر نتيجة التقدير في الجدول (9) بأن هناك إيجابية ودلالة إحصائية لكل معلمات التقدير. وتشير النتاجُ في المدى الطويل أو منطقة معلومات الأجل الطويل المتمثلة بالجزء الأول في المعادلة رخّ (24)، والتي يمكن من خلاله التوصّل إلى معادلة النكمل المشترك أي معادلة علاقة طويلة الأجل. وعند تفسيرنا لهذا

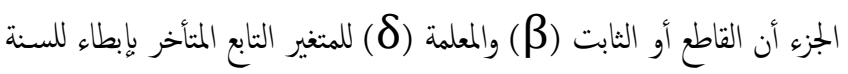
الواحدة ومعلمات الواحدة وتعبر عن أداء النموذج في الأجل الطويل، والذي يوضح طبيعة واتجاه العلاقات فيا بين كل من عجز الحساب الجاري ويز الموازنة العامة والفجوة الادخارية والتجارة، ولها معنى إحصائي وهي ضرورية لغرض فهم المعنى الاقتصادي للنموذج. ومن خلال تالك المؤشرات يمكن تحقيق التكمل المشترك فيا بين تلك المتغيرات إذا كان النموذج يتجه للتوازن في الأجل الطويل. الجدول 9. تيجة تثدير المملات في المدى التصير والطويل

\begin{tabular}{|c|c|c|c|c|}
\hline \multirow{2}{*}{$\begin{array}{c}\text { Dependent } \\
\text { variable d.CA }\end{array}$} & \multirow{2}{*}{\multicolumn{2}{|c|}{ PMG }} & \multirow{2}{*}{\multicolumn{2}{|c|}{ MG }} \\
\hline & & & & \\
\hline Independent variable & Coef. & الاحتماليبة & Coef. & الاحتمباكية \\
\hline PB & 0.54197 & 0.000 & -2.1552 & 0.26 \\
\hline SAV & 0.18429 & 0.000 & -0.05052 & 0.763 \\
\hline TRADE & -0.0415 & 0.000 & 2.7778 & 0.242 \\
\hline ECT & -0.518 & 0.000 & -1.574 & 0.004 \\
\hline d.PB & 0.059 & 0.450 & -0.022 & 0.937 \\
\hline d.SAV & -0.063 & 0.107 & -0.186 & 0.094 \\
\hline d.TRADE & 0.136 & 0.136 & 0.320 & 0.288 \\
\hline CONS & 3.497 & 0.000 & 29.483 & 0.304 \\
\hline
\end{tabular}




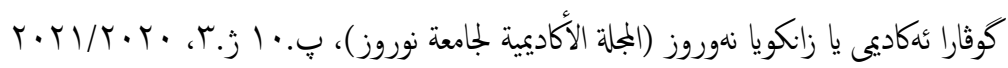

وتتم آلية عمل هذا الثأثير من خلال تقليل الاقتزاض وعبء الاقتراض، سواء كانت من الداخل أو الخارج وزيادة نسبه الصادرات، وبالتالي تنعكس هذه من مله

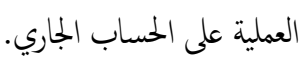

أما آلية عمل تأثير الحساب الجاري على الموازنة العامة في المدى الطويل من خلال

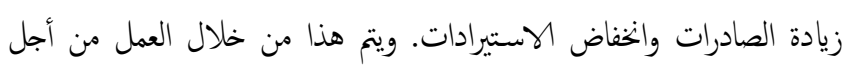
زيادة القدرة الإنتاجية وزيادة الصادرات وانخفاض الاستيرادات، وهذا بطبيعة الحال تؤثر على انخفاض الإنغاق على الاستيرادات.

\section{4. الاستناجات والمقترهات}

1.4 الاستناجات

لقد تم التوصل من خلال هذه الدراسة إلى جملة من الاستنتاجات، منها: تعدّ العلاقة بين العجز الملالي والفجوة الادخارية ويجز الحساب الجاري من أهم العلاقات بين المتغيرات الاقتصادية الكلية، وتعدّ هذه العلاقات النقطة المحورية للدراسات النظرية والتجريبة، وذلك لأن نتيجة وآثار تلك

العلاقات تظهر مباشرة على الكثير من جوانب الحياة الاقتصادية. تم قياس وتقدير العلاقة بين متغيرات الدراسة باستخدام طريقة العزوم المعتمة (PMM) و (GMARDL) المقدرة لـ (21) اقتصادات بلدان متطورة، وتشير الننائج إلى وجود علاقة قوية بين متغيرات الدراسة. ظهرت هذه النتيجة بعد إجراء وتطبيق الكثير من الطرق والمقارنة بينه من خلال مجموعة من المقايس ذات أهمية كبيرة في الدراسات القياسية الحديثة. إجراء التكامل المشترك على البيانات اللوحية، وتشير نتائج التقدير إلى وجود علاقة طويلة الأجل ومثانة النتائج المقدرة. وكانت النتائج ذات دلالة إحصائية وإيهابية لكل معلمات النموذج المقدر. وتشير النتاجُ بأن هناك تأثيرًا

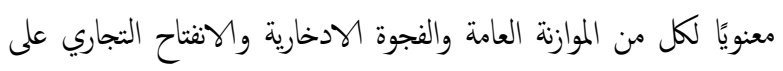
الحساب الجاري. وفي المقابل تتضح نناجُ التقدير في المدى القصير وتتعارض مع آثار تلك المتغيرات في المدى الطويل. وتتصف معامل تصحيح الخطأ بوجود إثارة السالبة ومعنوية وها شرطان الضروري والكلفي لإمكانية العودة إلى الوضع التوازني. ووفقًا لذلك، فإن سرعة التعديل في المدى القصير للوصول إلى مستوى التوازن على المدى الطويل هي 0.51 ٪ في تورس سنة. ومعامل تصحيح الخطأ تعبر عن النسبة المئوية من الخطأ من أخطاء الأجل القصير ، والتي يكن تصحيحها في واحدة الزمن من أجل العودة إلى
معامل تصحيح الخطأ بشرطين ها: إثارة السالبة ومعنوية للمعامل تصحيح الخطأ، وهذا بمعنى هناك تصحيح للخطأ أو حتى إمكانية العودة إلى الوضع التوازني. والخطوة الأخيرة كمة بالنسبة للموذجين هي تحديد واختيار النموذج النهائي الملائم ويتم هذا من خلال اختبار (Hausman)، ورغز وجود كثير من المؤشرات لقبول النموذج المفضل مثل قيمة الاحتالية وأيضًا قيمة المعلمات لكل من النموذجيين؛ إلا أن هذا الاختبار كمم لكي تستند إليه لاختيار النموذج الملائ. ويستند هذا الاختبار على فرضيتي العدم والبدياة، فإذا قبلنا فرضية العدم يكون النموذج

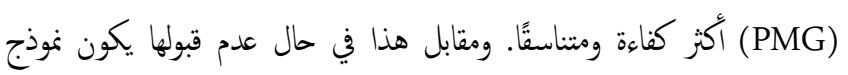
الثأثيرات الثابتة أكثر كفاءة وتنسيقًا.

\section{(Hausman Test) الجدول 10. نتاجُ اختبار}

hausman mg pmg, sigmamore

\begin{tabular}{|c|c|c|c|c|}
\hline & \multicolumn{2}{|c|}{ Coefficients -} & \multirow{3}{*}{$\begin{array}{c}(b-B) \\
\text { Difference }\end{array}$} & \multirow{3}{*}{$\begin{array}{c}\text { sqrt (diag (V_b-V_B ) ) } \\
\text { S.E. }\end{array}$} \\
\hline & (b) & (B) & & \\
\hline & $\mathrm{mg}$ & pmg & & \\
\hline $\mathrm{pb}$ & -2.155218 & .5419652 & -2.697183 & $5.69 \mathrm{e}+13$ \\
\hline sav & -.0505213 & .1842906 & -.2348119 & $4.97 e+12$ \\
\hline trade & 2.777859 & -.0415013 & 2.819361 & $7.07 e+13$ \\
\hline
\end{tabular}

Test: Ho: difference in coefficients not systematic

\begin{tabular}{|c|c|}
\hline $\operatorname{chi2}(3)=$ & $\begin{array}{l}=(b-B) \cdot\left[\left(V_{-} b-V_{-} B\right)^{\wedge}(-1)\right](b-B) \\
=0.00\end{array}$ \\
\hline Prob>chi2 = & 1.0000 \\
\hline
\end{tabular}

المصدر: - من إعداد الباحثين بناء على مخرجات STATA-15

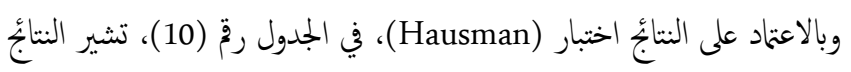
إلى أن القيمة الاحتمالية وهي (Prob>chi2 =1.000) أكبر من مستوى المعنوي (0.05)؛ ما يعني قبول فرضية العدم وهذا يعني أن النموذج (PMG) هو النموذج

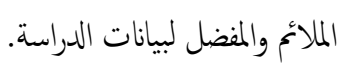
عند الوقوف على نتائُ العلاقة بين المتغيرات الثلاث في الفترتين القصيرة والطويلة نرى بأن هناك تأثيرات فيا بين المتغيرات الثلاث. وهذا يوحي بقدرة إمكانية تقدير وقياس وتحليل فرضية العجز الثلاثي بين كل من كجز الحساب الجاري والعجز في

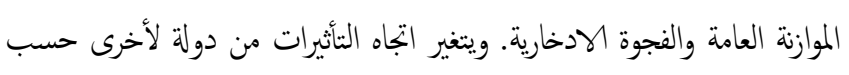
أنظمتها الاقتصادية والهيكل الاقتصادي، كما عرضنا نتائج اختبار لكل دولة على حدة في الجزء الخاص بالملاحق. ففي المدى الطويل هناك تأثير كبير للمتغيرات

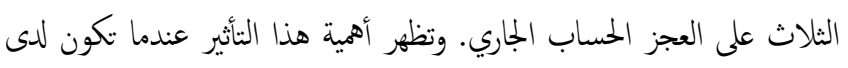
الدولة قدرة وإمكانية على توفير رأس المال اللازم لتغطية العملية الإنتاجية وزيادة الكفاءة الإنتاجية وإعادة التوازن إلى الموازنة العامة والفجوة الادخارية والتجارية. 


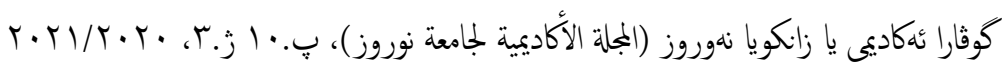

الاختلالات في المتغرات. إن تشخيص هذه الأسباب بمثابة الخطوة الأساسية لمعالجة تلك الاختلالات التي تحدث في التوازن، فثثلاً: تشخيص العوامل التي قد تؤثر على اختلال في بنود الموازنة العامة كلالايرادات والنفقات، ومن ثم تحديد العوامل التي تؤثر على الإيرادات كالضرائب والاختلال في قيمة العملة، ومن ثم التذبذب في نسبة الصادرات والواردات. فضلًا عن ذلك تحديد العوامل التي تؤثر على الادخار والاستثمار ومن ثم نسبة التجارة.. كل هذه العوامل التي تؤثر بشكل من الأشكال على الحساب الجاري. ومن خلال تلك الافتراضات والعوامل يككن أن تزال الآثار السلبية التي قد تحدث على يجز الحساب الجاري. إن التركيز الأساسي لهذه الدراسة كان على العجز الثلاثي؛ لذا من الممكن الاستفادة عمليًا من نتائجا وإدراجما إلى الواقع العملي حسب الهيكل الاقتصادي للدول. ومن المؤكد أن ينتغ من الجانب العملي خاصة فيه يتعلق بفكرة الادخار والاستثمار لأها تعد من العوامل الرئيسة في إعادة التوازن الداخلي، وبالتالي انغكاسه في التوازن الخارجي هذا من بجة، ومن جهةٍ أخرى بما أن هناك علاقة وثيقة بين كل من الادخار والاستثمار؛ لذا يجب أن تتوجه الأظظار إلى تقوية فكرة الادخار لدى المجتع، وهذا يعنى ارتفاع المستوى الثقافي لدى المجتمع تجاه فكرة الادخار (سياسة تشجيع الادخار

ينبغي على الدول ذات الاقتصادات المتنوعة انتهاج سياسة أكثر تكاملا، وذلك من خلال دراسة شاملة للمتغيرات الاقتصادية، ولن يقتصر الاهتخام فقط على جانب واحد والنظرة الأحادية للمتغيرات، لأنه من الممكن أن تتغير تلك التأثرات التي تتركها هذه العوامل في الدول المتقدمة بالنسبة للادول النامية أو المتخلفة.

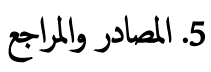

1.5

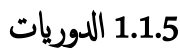

1. السواعي، خالد محمد والعزام، أنور أمد،، العجز التوهم في ظل المتغيرات النقدية والمالية

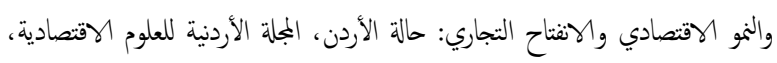
الجملد 2, 2015، العدد 2015.

2. العربية السعودية دراسات اقتصادية، السلسلة العلمية لممعية الاقتصاد السعودية، المجلد

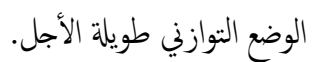

تشير أغلب نتاجُ الاختبارات إلى أن القيمة الاحتالية لاختبارات السكون للبيانات اللوحية أكبر من المستوى المعنوي (0.05) عند المستوى الأصلي، أي أن المتغيرات غير مستقرة عند المستوى الأصلي، عدا المتغير الثالث وهو متغير الفجوة الادخارية، وهو مستقر في المستوى الأصلي. وبعد أخذ الفرق الأول كانت ننائُ اختبارات جذر الوحدة للمتغيرات موضوع الدراسة أقل من المستوى المعنوي (0.05)؛ مما يعني سكون البيانات اللوحية عند أخذ الفرق الأول وإمكانية وجود علاقة طويلة الأجل

$$
\text { بين متغيرات موضوع الدراسة. }
$$

تشير الدراسة إلى المعنوية الإحصائية لبعض الدول، وعدم معنوية بعضها، وهذا يعود إلى أن نموذج الوندار التجميعي لا يعطي أي اعتبار للاختلافات الفردية بين الدول في حين كل دولة لها خصائص في سياستها المالية والاقتصادية تجاه هذه المتغيرات، وهذا بعد إجراء تلك التقديرات تح التوصل إلى أفضل طريقة لهذا النوع من البيانات، وهي طريقة (GMM) ونموذج تصحيح الأخطاء المستند إلى (PMG) لاستكشاف العلاقة بين عزز الحساب الجاري والعجز الملالي مع المتغيرات الأخرى مثل: أسعار الصرف والانقتاح النجاري.

Arellano- أظهرت نتائج التقدير قبول فرضية العدم من خلال اختبار Bond أظهرت النتأُج صلاحية الأدوات المستعلمة من خلال اختبار سارجان

(Sargan)

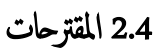

توصّلت الدراسة إلى جملة من المقترحات كمحاولة علمية جادة لمعالجة المشاكل في يجز الموازنة العامة وعجز الحساب الجاري والفجوة الادخارية، نذكر منها ما يلي: بما أن هناك إمكانية للعودة إلى الوضع التوازني كما توحي النتائُ من خلال تقدير النموذج القياسي، خاصة في الأجل القصير وأيضًا تحديد سرعة هذه العودة في المدى القصير للوصول إلى مستوى التوازن على المدى الطويل؛ لذا من المفيد أن نتزح العمل من أجل تصحيح مسار الاقتصادات ذات الطابع المشترك أو القريب من هذه الاقتصادات. البحث عن الأسباب والعوامل التي يكن أن تكون سبيًا في حدوث 
deregulation on milk price: A dynamic panel data approach, mpra, munich personal repee archive, 2018.

13. Fausten, D. K. (1989-90). Current and capital account interdependence. Journal of Post Keynesian Economics, 12 (2), 273-292.

14. Hilmi \& Fda triple deficit or twin divergence: a dynamic panel analysis, the International Journal of Economic and Social Research, Vol. 12, Year 12, No. 1, 2016.

\subsubsection{Books}

1. Bayramoglu \& Ozturk, Global Approaches in Financial Economics, Banking, and Finance, Contribution to Economic, Springer international publishing AG part of Springer Nature, 2018.

2. Damodar N. Gujarati (2003). basic econometrics, McGrawHiII/lrwin, a business unit of The McGraw-Hili Companies, Americas, New York.

3. William H. Greene, econometric analysis, eighth edition, pearson education, Inc, New York,2018.

4. Badi H. Baltgi, econometric analysis of panel data, third edition, John Wiley \& Sons Ltd, 2005.

5. Cheng Hsiao, analysis of panel data, combridge university press, econometric socity monographs, third edition 2014 .

\subsubsection{Website}

1. https://data.oecd.org/economy.htm\#profile-

International\%20trade

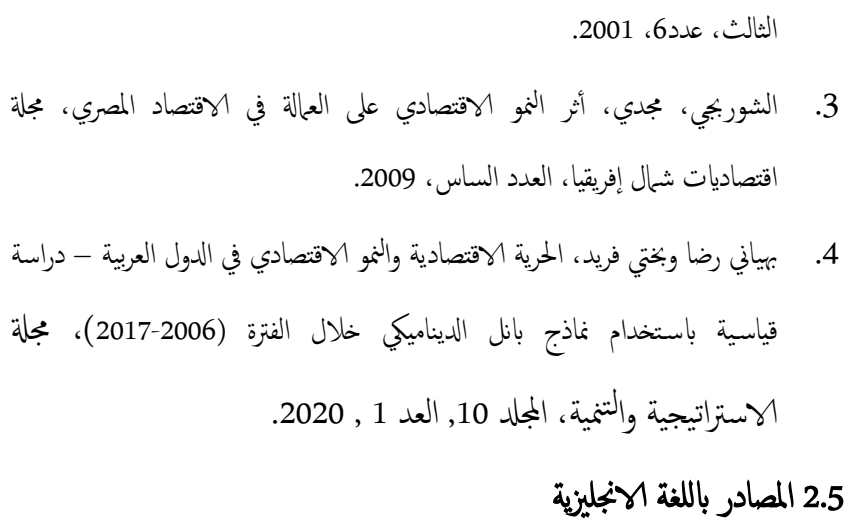

\subsection{Journals}

1. Emmanuel Anoruo and Sanjay Ramchander, Current Account and Fiscal Deficits Evidence from Five Developing Economies of Asia ,Journal of Asian Economics, Vol. 9, No. 3, 1998

2. Rosensweig\&Tallman:1993,590, Fiscal Policy and Trade Adjustment Are the Deficits Really Twins? Economic Inquiry, Vol. Xxxi, October 1993,580-594.

3. Olanrewaju.Raji, testing the validity of the triple deficit hypothesis for Nigeria, econometric research in finance, Vol.4 ,2019.

4. Miteza, Illir, Fiscal Deficits, Current Deficits and Investment: A Panel Causality Framework Of 20 Oecd Countries.

5. Tuck Cheong Tang Fiscal Deficit, Trade Deficit, and Financial Account Deficit: Triple Deficits Hypothesis with the U.S. Experience, Monash University, business and economics, 2014.

6. Coughlin, C, Pakko, M. R., \& Poole, W. (2006, April). How dangerous is the U.S. current account deficit? The Regional Economist.

7. Tang, T. C., \& Fausten, D. K. (2012). Current and capital account interdependence: an empirical test. International Journal of Business and Society, 13 (3).

8. Yuan-Ming Lee \& Kuan-Min Wang, Dynamic heterogeneous panel analysis of the correlation between stock prices and exchange rates, Economic Research-Ekonomska, Vol. 28, No. 1, Published by Taylor \& Francis,2015.

9. Nguyen Van Bon, Current Account and Fiscal Deficits Evidence of Twin Divergence from Selected Developing Economies of Asia, southeastasian journal of economics, 2014.

10. Jeffrey M. Wooldrindge, Applications of Generalized Method ofMoments Estimation, Journal of Economic Perspectives, Volume 15, Number 4, 2001.

11. Mnuel Arellano, some tests of specification for panel data: monte carlo evidence and an application to employment equations, the Review of Economic Studies, Vol.58, No.2,1991.

12. Fotis Panagiotis \& Polemis Michael, The impact of market 


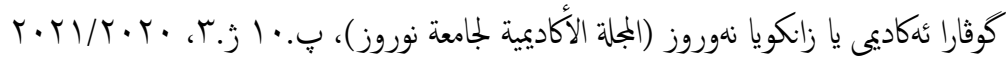

$$
\text { ثليجة نماذج المقدرة لكل من طريقة (PMG) و (MG) على التوالي }
$$

\begin{tabular}{|c|c|c|c|c|c|c|}
\hline \multicolumn{7}{|c|}{$\begin{array}{l}\text { Mean Group Estimation: Error Correction Form } \\
\text { (Estimate results saved as mg) }\end{array}$} \\
\hline D.ca & Coef. & Std. Err. & $\mathrm{z}$ & $P>|z|$ & [958 Conf & Interval] \\
\hline \multicolumn{7}{|c|}{$-2.155218 \quad 1.912941$} \\
\hline $\begin{array}{r}\text { pb } \\
\text { sav } \\
\text { trade }\end{array}$ & $\begin{array}{l}-2.155218 \\
-.0505213\end{array}$ & $\begin{array}{l}1.912941 \\
.1672073\end{array}$ & $\begin{array}{l}-1.13 \\
-0.30\end{array}$ & $\begin{array}{l}0.260 \\
0.763\end{array}$ & $\begin{array}{l}-5.904513 \\
-.3782415 \\
-1\end{array}$ & $\begin{array}{r}1.594078 \\
.2771989 \\
7.435607\end{array}$ \\
\hline \multicolumn{7}{|c|}{-2630986} \\
\hline ECT & -1.574125 & .5392249 & -2.92 & 0.004 & -2.630986 & -.5172636 \\
\hline $\begin{array}{l}\mathrm{pb} \\
\mathrm{D} 1\end{array}$ & -.0224427 & .2845843 & -0.08 & 0.937 & -.5802176 & .5353323 \\
\hline $\begin{array}{l}\text { sav } \\
\text { D1. }\end{array}$ & -.1858029 & .1108857 & -1.68 & 0.094 & -.4031348 & .031529 \\
\hline trade & & & & & & \\
\hline D1. & .3196814 & .3007238 & 1.06 & 0.288 & -.2697264 & .9090892 \\
\hline _oons & 29.48394 & 28.71108 & 1.03 & 0.304 & -26.78875 & 85.75662 \\
\hline
\end{tabular}

Pooled Mean Group Regression
(Estimate results saved as pmg)

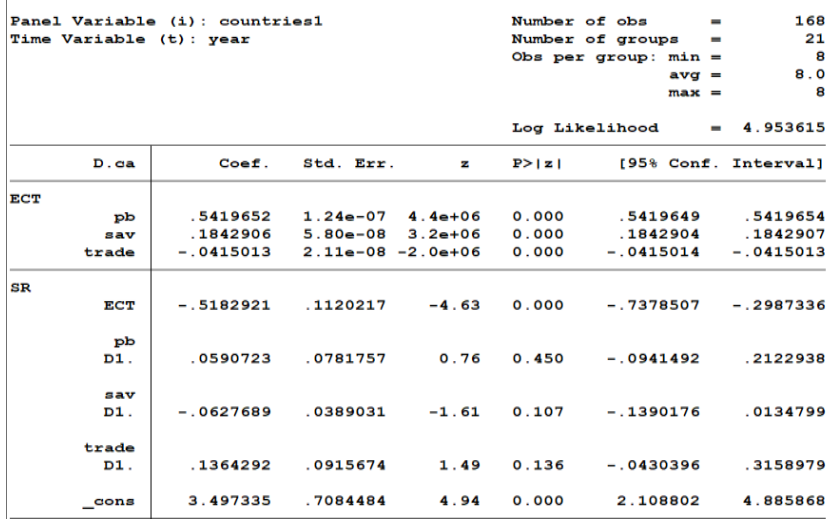

جداول المخاصة بالبيانات والمثغيرات موضوع المراسة

\begin{tabular}{|c|c|c|c|c|c|c|c|c|c|}
\hline IIرin' & $\mathbf{2 0 0 9}$ & $\mathbf{2 0 1 0}$ & $\mathbf{2 0 1 1}$ & $\mathbf{2 0 1 2}$ & $\mathbf{2 0 1 3}$ & $\mathbf{2 0 1 4}$ & $\mathbf{2 0 1 5}$ & $\mathbf{2 0 1 6}$ & $\mathbf{2 0 1 7}$ \\
\hline CA & & & & & & & & & \\
\hline Australia & -4.66 & -3.68 & -3.08 & -4.3 & -3.38 & -3.07 & -4.62 & -3.3 & -2.6 \\
\hline Canada & -2.91 & -3.57 & -2.72 & -3.53 & -3.14 & -2.32 & -3.5 & -3.1 & -2.8 \\
\hline Denmark & 3.47 & 6.56 & 6.59 & 6.28 & 7.76 & 8.92 & 8.25 & 7.77 & 7.76 \\
\hline Finland & 1.99 & 1.49 & -1.43 & -2.05 & -1.79 & -1.33 & -0.93 & -2.03 & -0.8 \\
\hline France & -0.55 & -0.63 & -0.86 & -0.97 & -0.51 & -0.96 & -0.37 & -0.49 & -0.7 \\
\hline Germany & 5.84 & 5.76 & 6.22 & 7.13 & 6.54 & 7.19 & 8.58 & 8.49 & 8.06 \\
\hline Israel & 3.23 & 3.61 & 1.51 & 0.34 & 2.99 & 4.05 & 4.97 & 3.27 & 2.27 \\
\hline Italy & -1.89 & -3.29 & -2.82 & -0.23 & 1.11 & 1.9 & 1.42 & 2.59 & 2.67 \\
\hline Japan & 2.78 & 3.87 & 2.09 & 1 & 0.9 & 0.79 & 3.12 & 3.93 & 4.2 \\
\hline Netherlands & 5.41 & 6.98 & 8.6 & 10.2 & 9.76 & 8.49 & 6.32 & 8.05 & 10.8 \\
\hline New Zealand & -2.27 & -2.24 & -2.8 & -3.92 & -3.09 & -3.11 & -2.71 & -2.04 & -5.5 \\
\hline Poland & -3.96 & -5.37 & -5.19 & -3.71 & -1.26 & -2.07 & -0.56 & -0.53 & 0.08 \\
\hline Spain & -4.09 & -3.66 & -2.72 & 0.1 & 2.04 & 1.69 & 2.03 & 3.17 & 2.68 \\
\hline Sweden & 5.96 & 5.88 & 5.48 & 5.51 & 5.18 & 4.49 & 4.11 & 3.54 & 3.07 \\
\hline United Kingdom & -3.33 & -3.17 & -1.76 & -3.43 & -4.76 & -4.72 & -4.91 & -5.21 & -3.5 \\
\hline United States & -2.58 & -2.88 & -2.87 & -2.64 & -2.08 & -2.08 & -2.24 & -2.29 & -2.3 \\
\hline Slovenia & -1.06 & -0.75 & -0.81 & 1.34 & 3.29 & 5.11 & 3.83 & 4.82 & 6.09 \\
\hline Hungary & -0.73 & 0.3 & 0.58 & 1.58 & 3.44 & 1.16 & 2.32 & 4.57 & 2.27 \\
\hline Belgium & 1.62 & 1.64 & -1.95 & -0.08 & 0.95 & 0.77 & 1.38 & 0.56 & 1.23 \\
\hline Iceland & -9.64 & -6.32 & -5.13 & -3.91 & 5.73 & 3.82 & 5.13 & 7.5 & 3.79 \\
\hline Portugal & -10.3 & -10.3 & -5.96 & -1.6 & 1.63 & 0.16 & 0.23 & 1.09 & 1.22 \\
\hline
\end{tabular}




\begin{tabular}{|c|c|c|c|c|c|c|c|c|c|}
\hline PB & $\mathbf{2 0 0 9}$ & $\mathbf{2 0 1 0}$ & $\mathbf{2 0 1 1}$ & $\mathbf{2 0 1 2}$ & $\mathbf{2 0 1 3}$ & $\mathbf{2 0 1 4}$ & $\mathbf{2 0 1 5}$ & $\mathbf{2 0 1 6}$ & $\mathbf{2 0 1 7}$ \\
\hline Australia & -5.56 & -4.46 & -4.62 & -2.89 & -2.84 & -2.33 & -2.43 & -1.84 & -0.8 \\
\hline Canada & -3.88 & -4.74 & -3.31 & -2.52 & -1.49 & 0.17 & -0.06 & -0.45 & -0.1 \\
\hline Denmark & -2.8 & -2.71 & -2.06 & -3.49 & -1.24 & 1.14 & -1.33 & -0.11 & 1.52 \\
\hline Finland & -2.48 & -2.53 & -1.02 & -2.16 & -2.52 & -2.99 & -2.42 & -1.73 & -0.7 \\
\hline France & -7.17 & -6.89 & -5.15 & -4.98 & -4.08 & -3.9 & -3.63 & -3.54 & -2.8 \\
\hline Germany & -3.15 & -4.38 & -0.88 & 0.01 & 0.04 & 0.58 & 0.94 & 1.18 & 1.24 \\
\hline Israel & -6.3 & -3.48 & -2.87 & -4.29 & -3.97 & -2.27 & -0.91 & -1.4 & -1.1 \\
\hline Italy & -5.12 & -4.24 & -3.59 & -2.95 & -2.85 & -2.95 & -2.55 & -2.41 & -2.4 \\
\hline Japan & -9.78 & -9.15 & -9.09 & -8.3 & -7.64 & -5.38 & -3.56 & -3.46 & -3 \\
\hline Netherlands & -5.09 & -5.25 & -4.43 & -3.92 & -2.93 & -2.15 & -2.02 & 0.02 & 1.26 \\
\hline New Zealand & -2.81 & -6.87 & -4.07 & -2.21 & -0.51 & 0.2 & 0.36 & 1.22 & 1.18 \\
\hline Poland & -7.25 & -7.4 & -4.88 & -3.74 & -4.18 & -3.65 & -2.62 & -2.37 & -1.5 \\
\hline Spain & -11.3 & -9.53 & -9.74 & -10.7 & -7.04 & -5.92 & -5.18 & -4.31 & -3 \\
\hline Sweden & -0.71 & -0.05 & -0.23 & -1.01 & -1.4 & -1.53 & 0 & 0.99 & 1.43 \\
\hline United Kingdom & -10.1 & -9.28 & -7.51 & -8.16 & -5.53 & -5.56 & -4.59 & -3.35 & -2.4 \\
\hline United States & -13.1 & -12.4 & -11 & -9.22 & -5.82 & -5.2 & -4.62 & -5.39 & -4.3 \\
\hline Slovenia & -5.81 & -5.6 & -6.63 & -3.99 & -14.6 & -5.51 & -2.85 & -1.93 & -0 \\
\hline Hungary & -4.69 & -4.39 & -5.19 & -2.27 & -2.54 & -2.75 & -1.97 & -1.76 & -2.4 \\
\hline Belgium & -5.43 & -4.09 & -4.33 & -4.32 & -3.13 & -3.06 & -2.41 & -2.36 & -0.7 \\
\hline Iceland & -9.48 & -9.46 & -5.41 & -3.61 & -1.78 & -0.07 & -0.79 & 12.43 & 0.54 \\
\hline Portugal & -9.87 & -11.4 & -7.66 & -6.18 & -5.11 & -7.36 & -4.45 & -1.89 & -3 \\
\hline & & & & & & & & & \\
\hline
\end{tabular}

\begin{tabular}{|c|c|c|c|c|c|c|c|c|c|}
\hline EXC & $\mathbf{2 0 0 9}$ & $\mathbf{2 0 1 0}$ & $\mathbf{2 0 1 1}$ & $\mathbf{2 0 1 2}$ & $\mathbf{2 0 1 3}$ & $\mathbf{2 0 1 4}$ & $\mathbf{2 0 1 5}$ & $\mathbf{2 0 1 6}$ & $\mathbf{2 0 1 7}$ \\
\hline Australia & 1.28 & 1.09 & 0.97 & 0.97 & 1.04 & 1.11 & 1.33 & 1.35 & 1.31 \\
\hline Canada & 1.14 & 1.03 & 0.99 & 1 & 1.03 & 1.11 & 1.28 & 1.33 & 1.3 \\
\hline Denmark & 5.36 & 5.62 & 5.37 & 5.79 & 5.62 & 5.61 & 6.73 & 6.73 & 6.6 \\
\hline Finland & 0.72 & 0.75 & 0.72 & 0.78 & 0.75 & 0.75 & 0.9 & 0.9 & 0.89 \\
\hline France & 0.72 & 0.75 & 0.72 & 0.78 & 0.75 & 0.75 & 0.9 & 0.9 & 0.89 \\
\hline Germany & 0.72 & 0.75 & 0.72 & 0.78 & 0.75 & 0.75 & 0.9 & 0.9 & 0.89 \\
\hline Israel & 3.93 & 3.74 & 3.58 & 3.86 & 3.61 & 3.58 & 3.89 & 3.84 & 3.6 \\
\hline Italy & 0.72 & 0.75 & 0.72 & 0.78 & 0.75 & 0.75 & 0.9 & 0.9 & 0.89 \\
\hline Japan & 93.6 & 87.8 & 79.8 & 79.8 & 97.6 & 106 & 121 & 109 & 112 \\
\hline Netherlands & 0.72 & 0.75 & 0.72 & 0.78 & 0.75 & 0.75 & 0.9 & 0.9 & 0.89 \\
\hline New Zealand & 1.6 & 1.39 & 1.27 & 1.23 & 1.22 & 1.21 & 1.43 & 1.44 & 1.41 \\
\hline Poland & 3.12 & 3.02 & 2.96 & 3.26 & 3.16 & 3.16 & 3.77 & 3.94 & 3.78 \\
\hline Spain & 0.72 & 0.75 & 0.72 & 0.78 & 0.75 & 0.75 & 0.9 & 0.9 & 0.89 \\
\hline Sweden & 7.65 & 7.21 & 6.49 & 6.78 & 6.51 & 6.86 & 8.44 & 8.56 & 8.55 \\
\hline United Kingdom & 0.64 & 0.65 & 0.62 & 0.63 & 0.64 & 0.61 & 0.66 & 0.74 & 0.78 \\
\hline United States & 1 & 1 & 1 & 1 & 1 & 1 & 1 & 1 & 1 \\
\hline Slovenia & 0.72 & 0.75 & 0.72 & 0.78 & 0.75 & 0.75 & 0.9 & 0.9 & 0.89 \\
\hline Hungary & 202 & 208 & 201 & 225 & 224 & 233 & 279 & 282 & 274 \\
\hline Belgium & 0.72 & 0.75 & 0.72 & 0.78 & 0.75 & 0.75 & 0.9 & 0.9 & 0.89 \\
\hline Iceland & 124 & 122 & 116 & 125 & 122 & 117 & 132 & 121 & 107 \\
\hline Portugal & 0.72 & 0.75 & 0.72 & 0.78 & 0.75 & 0.75 & 0.9 & 0.9 & 0.89 \\
\hline & & & & & & & & & \\
\hline
\end{tabular}

\begin{tabular}{|c|c|c|c|c|c|c|c|c|c|}
\hline تIائin' & $\mathbf{2 0 0 9}$ & $\mathbf{2 0 1 0}$ & $\mathbf{2 0 1 1}$ & $\mathbf{2 0 1 2}$ & $\mathbf{2 0 1 3}$ & $\mathbf{2 0 1 4}$ & $\mathbf{2 0 1 5}$ & $\mathbf{2 0 1 6}$ & $\mathbf{2 0 1 7}$ \\
\hline TRADE & & & & & & & & & \\
\hline Australia & 118 & 141.8 & 143 & 129 & 124 & 111 & 100 & 114.3 & 117 \\
\hline Canada & 101 & 106.5 & 110 & 108 & 109 & 108 & 100 & 98.9 & 102 \\
\hline Denmark & 96.1 & 98.4 & 96.6 & 97.1 & 98.1 & 99.6 & 100 & 99.9 & 99.7 \\
\hline Finland & 101 & 98.6 & 97 & 96 & 96.7 & 97.4 & 100 & 100 & 99.4 \\
\hline France & 98.6 & 97.2 & 94.9 & 94.6 & 95.7 & 96.9 & 100 & 101 & 99.7 \\
\hline Germany & 100 & 98.2 & 95.6 & 95.5 & 96.5 & 97.7 & 100 & 101.7 & 101 \\
\hline Israel & 91.6 & 89.8 & 86.8 & 89.3 & 91.3 & 92.3 & 100 & 104 & 104 \\
\hline Italy & 102 & 97.6 & 95.1 & 93.7 & 95.3 & 97.7 & 100 & 103.3 & 102 \\
\hline Japan & 108 & 102 & 94.2 & 93.1 & 92.2 & 91.7 & 100 & 105.4 & 101 \\
\hline Netherlands & 102 & 100.1 & 98.6 & 98.6 & 99 & 98.9 & 100 & 100.6 & 100 \\
\hline New Zealand & 88.4 & 95.3 & 96.7 & 92.5 & 103 & 103 & 100 & 103.1 & 108 \\
\hline Poland & 99 & 97.6 & 96.1 & 94.9 & 95.9 & 97.7 & 100 & 100.5 & 101 \\
\hline Spain & 107 & 103.7 & 100 & 98.9 & 100 & 99.5 & 100 & 100.4 & 99.6 \\
\hline Sweden & 99.8 & 99.4 & 98.7 & 98.8 & 99 & 99.3 & 100 & 100.3 & 99.4 \\
\hline United Kingdom & 94.9 & 96.1 & 94.6 & 94.9 & 96.7 & 98.5 & 100 & 100.9 & 100 \\
\hline United States & 96.1 & 94.9 & 93.7 & 94.3 & 95.8 & 96.6 & 100 & 101.6 & 102 \\
\hline Slovenia & 104 & 99.4 & 98 & 97 & 97.8 & 98.7 & 100 & 100.8 & 100 \\
\hline Hungary & 101 & 100.5 & 99.1 & 98.2 & 98.7 & 99.3 & 100 & 100.7 & 100 \\
\hline Belgium & 102 & 99.7 & 98.3 & 98.3 & 98.7 & 99 & 100 & 100.6 & 100 \\
\hline Iceland & 93.8 & 98.1 & 95.4 & 92.5 & 90.7 & 93.7 & 100 & 102.4 & 104 \\
\hline Portugal & 96.8 & 95.5 & 93.7 & 94.3 & 96 & 96.9 & 100 & 101.6 & 101 \\
\hline
\end{tabular}

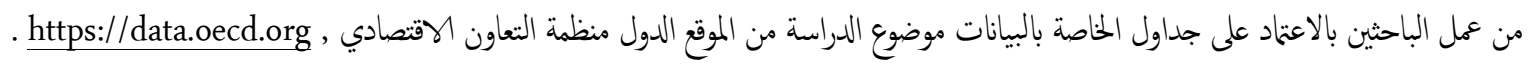
تنظيم وترتيب بعض البيانات واستخراج النتائُ النهائية لتلك البيانات مثل بيانات عن المتغير (الفجوة الادخارية) من خلال طرح الاستثمار من الادخار 